\title{
Hydrodynamic fluctuations in quasi-two dimensional diffusion
}

\author{
Raúl P. Peláez, ${ }^{1}$ Florencio Balboa Usabiaga, ${ }^{2,3}$ Sergio Panzuela, ${ }^{4}$ \\ Qiyu Xiao, ${ }^{2}$ Rafael Delgado-Buscalioni, ${ }^{1}$ and Aleksandar Donev ${ }^{2}$, \\ ${ }^{1}$ Departamento de Fúsica Teórica de la Materia Condensada, \\ Universidad Autónoma de Madrid, Madrid, Spain \\ ${ }^{2}$ Courant Institute of Mathematical Sciences, \\ New York University, New York, NY, USA \\ ${ }^{3}$ Center for Computational Biology, Flatiron Institute, \\ Simons Foundation, New York, NY, USA \\ ${ }^{4}$ Departamento de Fúsica Teórica de la Materia Condensada, \\ and Institute for Condensed Matter Physics, IFIMAC, \\ Universidad Autónoma de Madrid, Madrid, Spain
}




\begin{abstract}
We study diffusion of colloids on a fluid-fluid interface using particle simulations and fluctuating hydrodynamics. Diffusion on a two-dimensional interface with three-dimensional hydrodynamics is known to be anomalous, with the collective diffusion coefficient diverging like the inverse of the wavenumber. This unusual collective effect arises because of the compressibility of the fluid flow in the plane of the interface, and leads to a nonlinear nonlocal convolution term in the diffusion equation for the ensemble-averaged concentration. We extend the previous hydrodynamic theory to account for a species/color labeling of the particles, as necessary to model experiments based on fluorescent techniques. We study the magnitude and dynamics of density and color density fluctuations using a novel Brownian dynamics algorithm, as well as fluctuating hydrodynamics theory and simulation. We find that hydrodynamic coupling between a single tagged particle and collective density fluctuations leads to a reduction of the long-time self-diffusion coefficient, even for an ideal gas of non-interacting particles. This unexpected finding demonstrates that density functional theories that do not account for thermal fluctuations are incomplete even for ideal systems. Using linearized fluctuating hydrodynamics theory, we show that for diffusion on a fluid-fluid interface, nonequilibrium fluctuations of the total density are small compared to the equilibrium fluctuations, but fluctuations of color density are giant and exhibit a spectrum that decays as the inverse cubed power of the wavenumber. We confirm these predictions through Brownian dynamics simulations of diffusive mixing with two indistinguishable species. We also examine nonequilibrium fluctuations in systems with two-dimensional hydrodynamics, such as thin smectic films in vacuum. We find that nonequilibrium fluctuations are colossal and comparable in magnitude to the mean, and can be accurately modeled using numerical solvers for the nonlinear equations of fluctuating hydrodynamics.
\end{abstract}

*Electronic address: donev@courant.nyu.edu 


\section{INTRODUCTION}

Diffusion of colloidal particles confined to two-dimensional surfaces is a key transport mechanism in several contexts of technological and biological significance. Colloidal particles can spontaneously absorb on fluid-fluid interfaces and stabilize Pickering emulsions [1]. The transverse diffusion of proteins embedded in lipid bilayers controls their biological function [2]. In man-made colloidal suspensions, colloidal particles can be confined to primarily diffuse in a plane by walls [3] or electrostatic forces [4]. While much is understood about complex fluid-fluid interfaces [1], fundamental questions about diffusive transport at interfaces remain unanswered [5].

Bulk diffusion of particles in liquids is well-known to be controlled by hydrodynamics, and diffusion on interfaces is no exception. While the diffusion of colloids and polymers on a fluid-fluid interface has been studied theoretically since the 1970s [6, 7], collective diffusion in a monolayer of colloidal particles confined to a fluid-fluid interface has only recently been explored in some detail [8-10]. These recent studies have shown that collective diffusion on interfaces is anomalous, with the short-time collective diffusion coefficient diverging as the inverse of the wavenumber. This unexpected finding has prompted re-examination of previous experimental results [3, 11, 12, and it is plausible that the effect may have been overlooked in a number of other prior experimental and theoretical works as well. In addition to the practical implications, the anomalous character of collective diffusion on interfaces brings into question the very applicability of Fick's law even at macroscopic scales, which brings into question fundamental assumptions in the phenomenological foundation of nonequilibrium thermodynamics.

The physical origin of the anomalous collective diffusion has already been elucidated in prior work by others [8 10]. Stiff normal forces are required to confine the colloidal particles to a plane. These forces will propagate momentum to the plane via the incompressible solvent. In the plane, the resulting hydrodynamic drag created by one particle acts like a flow source that induces an effective repulsive force on the other particles. This results in strong displacement correlations which are the origin of the anomalous collective diffusion. A new interpretation of this phenomena is obtained if one focuses on the in-plane dynamics. In the plane, the field of diffusive displacements appears to be compressible due to the momentum source associated with each confined particle. In the limit of strict con- 
finement, the flow acts as a compressible two-dimensional cut through an incompressible three-dimensional flow field. Because of this apparent compressibility, the Brownian motion of the particles creates an osmotic pressure proportional to $k_{B} T$, which propagates via the fluid inducing long-ranged repulsive interactions. This long-ranged repulsion decays slowly with the particle-particle distance, and dramatically accelerates collective diffusion at large scales. Furthermore, contrary to what was previously thought [13, 14, these long-ranged correlations also appreciably modify the single-particle diffusion, as we show in this work.

Previous work on collective diffusion of colloids on fluid-fluid interfaces [8-10] has focused on the ensemble average. In particular, in [9] the authors use the ensemble-averaged equations from the Dynamic Density Functional Theory with Hydrodynamic Interactions (DDFT-HI) developed in [15]. Our focus here is on the fluctuations around the ensemble average, i.e., on the equations for the evolution of a particular instance (trajectory) of diffusive processes on interfaces. We use the fluctuating DDFT-HI developed in [15], which is closely-related to fluctuating hydrodynamics (FHD) [16]. The (formal) nonlinear equations of FHD are challenging to interpret [15, 17], but, at the same time, they are more physically transparent than the ensemble-averaged equations, and do not require closures for noninteracting particles [15]. Furthermore, as we will see later, the fluctuating DDFT-HI equations are a very useful tool for constructing linear-time Brownian Dynamics (BD) algorithms.

Understanding the magnitude and the dynamics of density fluctuations is crucial for several reasons. First, in actual experiments one observes individual instances, not the ensemble average. While in many systems typical instances are quite similar to the average, i.e., the fluctuations are small compared to the mean, this is not always the case. It is well-known that nonequilibrium fluctuations in diffusive processes are, rather generally, much larger in magnitude than equilibrium fluctuations, and are also long ranged [18]. Experiments in microgravity have measured these "giant fluctuations" in three dimensions, and shown that nonequilibrium diffusive fluctuations are correlated over macroscopic distances [19, 20]. For diffusion in two-dimensional systems such as thin smectic films in vacuum [21], linearized FHD predicts that the magnitude of the nonequilibrium fluctuations becomes comparable to the mean [22]. The appearance of such "colossal fluctuations" implies that the ensemble average is no longer informative, since each instance looks very different from the mean. It is therefore essential to understand whether individual instances of diffusive mixing processes on fluid-fluid interfaces evolve similarly to the ensemble average. Second, fluctuations can be 
measured in experiments and reveal information about the underlying microscopic mechanism of diffusion. For diffusion in bulk three-dimensional liquids or in truly two-dimensional liquids (e.g., a hard-disk fluid), the ensemble average strictly follows the familiar Fick's law [16]. This means that the ensemble-averaged concentration in a system where the diffusing particles are strongly correlated by hydrodynamics (e.g., two-dimensional thin smectic films [23]), will look indistinguishable from the ensemble average in a system where the diffusing particles are uncorrelated (e.g., diffusion in a solid). But if one examines the magnitude of the nonequilibrium fluctuations, the hydrodynamic correlations are revealed through an unexpected power-law dependence of the structure factor on the wavenumber [22]. Third, collective fluctuations can couple bi-directionally to the motion of each particle and therefore renormalize transport coefficients. For example, the fluctuating hydrodynamic theory developed in [24] shows that collective density fluctuations reduce the effective diffusion coefficient of a tagged particle in a dense system of uncorrelated Brownian soft spheres. In this paper we will show that a similar effect exists even for an ideal gas of non-interacting but hydrodynamically-correlated particles diffusing on a fluid-fluid interface.

In this paper we study the ensemble average and fluctuations of the density of spherical colloidal particles confined to diffuse on a fluid-fluid interface. We closely mimic the physical setting used in prior studies [8 10], and make a number of strong simplifying assumptions:

1. We assume that the interface is perfectly flat and that two fluids have the same viscosity; the case of unequal viscosity simply amounts to taking the arithmetic average of the two viscosities [6, 7].

2. In order to isolate the role of hydrodynamics from the role of other direct interactions such as capillary forces or steric/electrostatic repulsion, we focus on an ideal gas of non-interacting spherical colloids [10]. While such an idealized system could not be studied experimentally, it is a natural candidate for testing existing and developing new theories. Furthermore, light scattering observational data for colloids at a fluid interface support predictions made for an ideal gas of particles [10].

3. We assume that the colloids are strictly confined to the interface by a strong confining force in the $z$-direction, i.e., they cannot leave the $x-y$ plane. In reality, the confinement would be partial, for example, a laser sheet may provide a harmonic confining potential in the $z$ direction. However, prior work [13, 14] has shown that partial con- 
finement only changes the results for larger wavenumbers (i.e., for wavelengths smaller than or comparable to the range of movement in the $z$ direction), and does not affect the anomalous diffusion in the plane. At the same time, we will show here that the case of strict confinement can be simulated much more efficiently using a two-dimensional Brownian dynamics algorithm.

4. We use a minimal far-field description of the hydrodynamics, as used in prior work by others [8 10]. Such a Rotne-Prager approximation is quantitatively accurate only for dilute suspensions, and we may question its usefulness in studying a collective effect that is dominant at larger packing densities. Nevertheless, as already mentioned, the anomalous collective diffusion arises because of a long-ranged (far-field) repulsion $\sim k_{B} T$. We therefore believe that short-ranged (near-field) corrections to the hydrodynamics will not qualitatively change the phenomena studied here.

While our focus is diffusion on a fluid-fluid interface, which we will refer to as Quasi2D (abbreviated q2D) diffusion, we will contrast Quasi2D diffusion to diffusion in truly twodimensional systems such as thin films in vacuum [21], which we will refer to as True2D (abbreviated t2D). Even though the diffusion is constrained to a two-dimensional plane in both cases, the hydrodynamics is essentially three-dimensional in Quasi2D but it is twodimensional in True2D. Our computational and analytical tools can easily be extended to other types of hydrodynamics. For example, it is believed that lateral diffusion in lipid membranes [2] or thin smectic films in air [23] can be described using a hydrodynamic model first proposed by Saffman. The (2+1)-dimensional $((2+1)$ D for short) Saffman model has already been combined with linearized fluctuating hydrodynamics in [22, but an experimental confirmation of the predictions of the theory is still lacking.

We begin by summarizing the relevant aspects of fluctuating DDFT-HI theory [15] in Section II, and then use DDFT-HI to re-examine some observations about the unusual nature of Quasi2D diffusion made in prior work by others [8-10]. In Section III] we develop and validate a novel algorithm for performing Brownian Dynamics with Hydrodynamic Interactions (BD-HI) in two dimensions (BD-2D). Our pseudo-spectral algorithm scales linearly with the number of particles and can efficiently be implemented on Graphical Processing Units (GPUs). We use the BD-2D algorithm to perform large scale simulations in Quasi2D and True2D. In particular, in Section IV we study the evolution of the ensemble average in 
free diffusion in Quasi2D. Our studies confirm prior results, but we also perform a new type of numerical experiment by coloring (labeling) the particles with two colors (species labels). We clarify several aspects of the anomalous diffusion by studying the evolution of the ensemble average not just for the total density but also for color (species) density. In Section $\mathrm{V}$ we study the effect of collective fluctuations on the long-time self-diffusion coefficient of a tagged (tracer) particle, and quantify how density affects the long-time self diffusion in Quasi2D systems. In Section VI we study the magnitude and dynamics of density and color density fluctuations in True2D and Quasi2D. Finally, we offer some Conclusions and topics for future work.

\section{FLUCTUATING DYNAMIC DENSITY FUNCTIONAL THEORY WITH HY- DRODYNAMIC INTERACTIONS}

In this section we review some prior results regarding colloidal diffusion in quasi twodimensional (Quasi2D) geometry. Our Dynamic Density Functional Theory with Hydrodynamic Information (DDFT-HI) formulation is based on several prior works, and in particular [9]. At the same time, we account for fluctuations using the approach proposed in [15], and our presentation differs in several important aspects from prior work even though the results we derive in this section are already known.

\section{A. From Partial to Strict Confinement}

As a preamble to this section we consider a physical setup where colloids immersed in an unbounded three-dimensional fluid are confined to remain near the plane $z=0$ by a strong confining potential, as could be experimentally done using using a laser sheet trap. Due to the fluid incompressibility, part of the momentum introduced by a confinement force applied to one colloid (in the normal direction) will spread over the plane according to the Oseen tensor. As we show next, in the $z=0$ plane, the resulting hydrodynamic drag acts like a flow-source which tends to expel other particles around it because the generated velocity field has a positive divergence.

In this paper we consider the limit of infinitely strong confining forces. In this limit, it is not difficult to prove [13] that the effective hydrodynamic drag is proportional to 
the divergence of the hydrodynamic mobility evaluated in the plane. To see this, let us consider non-interacting particles of radius $a$ confined to the $x-y$ plane by a quadratic potential $U(z)=\left(k_{s} / 2\right) z^{2}$. At a given temperature $T$ the typical displacement of the particles around the confining plane $z=0$ is $\delta=\left(k_{B} T / k_{s}\right)^{1 / 2}$. The Ito equations of Brownian Dynamics with Hydrodynamic Interactions (BD-HI) for the (correlated) positions $\boldsymbol{Q}(t)=\left\{\boldsymbol{q}_{1}(t), \ldots, \boldsymbol{q}_{N}(t)\right\}$ of $N$ spherical colloidal particles, where $\boldsymbol{q}_{i}=\left(x_{i}, y_{i}, z_{i}\right) \in \mathbb{R}^{3}$, have the general form

$$
d \boldsymbol{Q}=\boldsymbol{M} \boldsymbol{F} d t+\left(2 k_{B} T \boldsymbol{M}\right)^{\frac{1}{2}} d \boldsymbol{B}+k_{B} T\left(\partial_{\boldsymbol{Q}} \cdot \boldsymbol{M}\right) d t
$$

where $\boldsymbol{B}(t)$ is a vector of Brownian motions, and $\boldsymbol{F}=-\partial_{\boldsymbol{Q}} U$ are the forces arising from the confining potential; other forces (such as excluded volume interactions) could be added in this discussion without loss of generality. The symmetric positive semidefinite (SPD) mobility matrix $\boldsymbol{M}(\boldsymbol{Q}) \succeq \mathbf{0}$ encodes all of the information about hydrodynamic interactions (correlations) - we will discuss its form in more detail later. Within the pairwise RotnePrager approximation for $\boldsymbol{M}$, we have $\partial_{\boldsymbol{Q}} \cdot \boldsymbol{M}=0$ because of the incompressibility of the (three-dimensional) flow.

Prior work [8-10] focusing on strict confinement has simply started from the equations (1) with the $z$ component ignored, but these equations can be derived precisely by taking the limit $k_{s} \rightarrow \infty$, as we explain next. Two distinct prior works [13, 14] have examined the transition from partial to strict confinement. The results of these prior studies have shown that partial confinement only changes the results for wavelengths smaller than or comparable to the range of movement in the $z$ direction. For larger wavelengths the system behaves as if the confinement is strict.

For infinitely strong confinement $k_{s} \rightarrow \infty$ and $\delta \rightarrow 0$, particles move strictly in the plane, with $z=0$. It is well known how to take a limit of overdamped Langevin equations in the presence of a stiff confining potential. The general theory for this is quite complicated [2528] because it involves metrics/curvatures of the constraint manifold and projected mobility matrices, but in our case taking this limit is trivial because of the following two generic properties. The first important property is that for particles strictly confined to the $(x, y)$ plane the mobility matrix

$$
\boldsymbol{M}=\left[\begin{array}{cc}
\boldsymbol{M}^{\|} & \boldsymbol{M}^{\|, \perp} \\
\left(\boldsymbol{M}^{\|, \perp}\right)^{T} & \boldsymbol{M}^{\perp}
\end{array}\right]=\left[\begin{array}{cc}
\boldsymbol{M}^{\|} & \\
& \boldsymbol{M}^{\perp}
\end{array}\right]
$$


has a block-diagonal structure in which the $z$ direction is decoupled to the $(x, y)$ directions, where we have ordered the parallel or $(x, y)$ degrees of freedom before the perpendicular or $z$ degrees of freedom. This follows from symmetry arguments: Applying a force parallel to the plane cannot induce motion normal to the plane because both sides are symmetric; similarly, normal forces cannot induce a velocity in the plane. Another important property is that there is no free energy gradient in the plane due to the confinement because the confining potential is uniform in the $(x, y)$ plane. This implies that there is no entropic gradient in the plane coming from non-uniform confinement to the plane.

Combining these two properties leads to the trivial conclusion that one can simply ignore the $z$ component of the original equations and just take the $x-y$ component of the deterministic terms in the equation. Specifically, the limiting equation is identical to (1) but with $\boldsymbol{q}_{i}=\left(x_{i}, y_{i}\right) \in \mathbb{R}^{2}$ and $\boldsymbol{F}_{i}$ now being the $(x, y)$ components of the particle positions and applied forces, and $\boldsymbol{M}$ replaced by the $(x, y)$ diagonal block $\boldsymbol{M}^{\|}$of the three-dimensional mobility matrix,

$$
d \boldsymbol{Q}^{\|}=\boldsymbol{M}^{\|} \boldsymbol{F}^{\|} d t+\left(2 k_{B} T \boldsymbol{M}^{\|}\right)^{\frac{1}{2}} d \boldsymbol{B}^{\|}+k_{B} T\left(\partial_{\boldsymbol{Q}^{\|}} \cdot \boldsymbol{M}^{\|}\right) d t
$$

where $\boldsymbol{Q}_{\|}$denotes the positions in the plane.

It is instructive to explain how the nonzero stochastic drift term $k_{B} T\left(\partial_{\boldsymbol{Q}_{\|}} \cdot \boldsymbol{M}^{\|}\right)$, which is necessary for time reversibility (detailed balance) of the limiting dynamics (2), arises in the strict confinement limit $\delta / a \rightarrow 0$. For strong confinement, the particles' height fluctuates rapidly around the $z=0$ plane and thus reach a quasi-steady equilibrium state in the $z$ direction. This means that the probability distribution for observing the particles in a given configuration factorizes in the form

$$
P(\boldsymbol{Q}, t) \approx P_{\|}\left(\boldsymbol{Q}_{\|}, t\right) \prod_{i=1}^{N} P_{\perp}\left(z_{i}\right)
$$

where $P_{\perp}(z) \sim \exp (-\beta U(z))$ is the Gibbs-Boltzmann distribution in the perpendicular direction. The parallel velocity of one particle at $z_{1}=0$ due to the drag created by a second particle rapidly fluctuating in the $z$ direction, time-averaged over the fast $z$ motion of the particle at $z_{2}$, is $\boldsymbol{u}_{1}^{\|}=\int d z_{2} P_{\perp}\left(z_{2}\right) M_{12}^{\|, \perp} F_{2}^{\perp}$, where $F_{2}^{\perp}=-\partial_{z_{2}} U\left(z_{2}\right)$ and the component $\boldsymbol{M}_{12}^{\|, \perp}$ of the mutual mobility measures the hydrodynamic coupling between perpendicular forces and parallel flows. Since $F^{\perp}\left(z_{2}\right) P_{\perp}\left(z_{2}\right)=\left(k_{B} T\right) \partial_{z_{2}} P_{\perp}\left(z_{2}\right)$, an integration by parts 
reveals that

$$
\boldsymbol{u}_{1}=\int d z_{2} \boldsymbol{M}_{12}^{\|, \perp} \partial_{z_{2}} P_{\perp}\left(z_{2}\right)=-\left(k_{B} T\right) \int d z_{2} P_{\perp}\left(z_{2}\right) \partial_{z_{2}} \boldsymbol{M}_{12}^{\|, \perp}
$$

In the limit of strict confinement $P_{\perp}(z) \rightarrow \delta(z)$ becomes a Dirac $\delta$ function enforcing $z_{1}=z_{2}=0$. Therefore one concludes that

$$
\boldsymbol{u}_{1}^{\|} \approx-\left(k_{B} T\right)\left(\partial_{z_{2}} \boldsymbol{M}_{12}^{\|, \perp}\right)_{z_{1}=z_{2}=0}=\left(k_{B} T\right)\left(\partial_{\boldsymbol{q}_{2}^{\|}} \cdot \boldsymbol{M}_{12}^{\|}\right)_{z_{1}=z_{2}=0},
$$

where we have used the incompressibility of the three-dimensional flow to conclude that the

pairwise mobility satisfies $\left(\partial_{\boldsymbol{q}_{2}^{\|}} \cdot \boldsymbol{M}_{12}^{\|}\right)+\partial_{z_{2}} \boldsymbol{M}_{12}^{\|, \perp}=\mathbf{0}$. This demonstrates that the strong confinement leads to an additional stochastic drift velocity in the plane of the form

$$
\boldsymbol{u}^{\|}=\left(k_{B} T\right) \partial_{\boldsymbol{Q}_{\|}} \cdot \boldsymbol{M}^{\|}
$$

which is the cause of the unusual collective effects on diffusion in the plane. For simplicity of notation, henceforth we will use the same generic (1) for both motion in three and two dimensions, and distinguish only when necessary.

\section{B. DDFT-HI Theory}

We begin by summarizing some key results of DDFT-HI, previously obtained in [15], by starting from the BD-HI equation (1). We assume here that the limit of strict confinement has already been taken if one is considering a suspension confined to a plane, so that particles diffuse in the plane only. In this paper we focus on an ideal gas of hydrodynamicallyinteracting particles, i.e., we take $\boldsymbol{F}=0$. In this work we consider periodic boundary conditions only; however, the formalism is rather general. For simplicity in this section we assume the system is unbounded (i.e., in free space). Periodicity can be handled by using the free-space formulation and summing over periodic images; this is most easily done numerically in Fourier space and is transparently handled in our algorithm by using the Fast Fourier Transform (FFT).

To leading order in the far-field, $\boldsymbol{M}(\boldsymbol{Q})$ is given by the pairwise approximation [15]

$$
\forall(i, j): \quad \boldsymbol{M}_{i j}\left(\boldsymbol{q}_{i}, \boldsymbol{q}_{j}\right)=\boldsymbol{\mathcal { R }}\left(\boldsymbol{q}_{i}, \boldsymbol{q}_{j}\right)
$$


where the tensor $\mathcal{R}\left(\boldsymbol{r}, \boldsymbol{r}^{\prime}\right)$ is a symmetric positive-semidefinite (SPD) hydrodynamic kernel that depends on the geometry and boundary conditions ${ }^{1}$. For particles very far apart $\mathcal{R}$ approaches the Green's function (also called Oseen tensor) for Stokes flow in the specified geometry with the specified boundary conditions. A commonly-used model of the hydrodynamic kernel, suitable in an unbounded three-dimensional system, is the Rotne-PragerYamakawa (RPY) kernel [29]. Because we assume that (3) holds even if $i=j$ (which is true for the RPY tensor), the (short-time) self-diffusion tensor of a particle with position $\boldsymbol{r}$ is

$$
\chi(\boldsymbol{r})=\left(k_{B} T\right) \mathcal{R}(\boldsymbol{r}, \boldsymbol{r})
$$

We consider here a translationally-invariant and isotropic system, for which symmetry dictates the form

$$
\mathcal{R}\left(\boldsymbol{q}_{i}, \boldsymbol{q}_{j}\right)=\mathcal{R}\left(\boldsymbol{r}=\boldsymbol{q}_{i}-\boldsymbol{q}_{j}\right)=f(r) \boldsymbol{I}+g(r) \frac{\boldsymbol{r} \otimes \boldsymbol{r}}{r^{2}}
$$

where $g(0)=0$ and $\otimes$ denotes a diadic product, $\boldsymbol{r} \otimes \boldsymbol{r} \equiv \boldsymbol{r} \boldsymbol{r}^{T}$ where superscript $T$ denotes a transpose. The majority of our discussion is focused on particles on a flat two-dimensional fluid-fluid interface, in an otherwise unbounded three-dimensional fluid. We refer to this setup as quasi two-dimensional (Quasi2D or q2D) diffusion $\left(\boldsymbol{r} \in \mathbb{R}^{2}\right)$, to be contrasted with true two-dimensional (True2D or t2D) diffusion $\left(\boldsymbol{r} \in \mathbb{R}^{2}\right)$ in a thin liquid film suspended in vacuum, or true three-dimensional (True3D or $\mathrm{t} 3 \mathrm{D}$ ) diffusion in a bulk liquid $\left(\boldsymbol{r} \in \mathbb{R}^{3}\right)$. For True3D systems, the (short-time) self diffusion of the particles obeys the Stokes-Einstein relationship

$$
\boldsymbol{\chi}(\boldsymbol{r})=\left(k_{B} T\right) \mathcal{R}(\mathbf{0})=\chi_{0} \boldsymbol{I}=\frac{k_{B} T}{(6 \pi \eta a)} \boldsymbol{I},
$$

where $\eta$ is the fluid viscosity ${ }^{2}$ and $a$ is the hydrodynamic radius of the colloidal particles. Furthermore, for $r \gg a$ the hydrodynamic kernel approaches the three-dimensional Oseen tensor,

$$
f(r \gg a) \approx g(r \gg a) \approx \frac{1}{8 \pi \eta r} .
$$

The subscript zero in $\chi_{0}$ emphasizes that this is the short-time self diffusion coefficient, which can in principle be different from the long-time self diffusion coefficient of the particles

\footnotetext{
${ }^{1}$ Note that we have adjusted slightly the notation in [15] to extract $\left(k_{B} T\right)$ outside of $\mathcal{R}$, so that $\mathcal{R}$ only contains hydrodynamic information and involves the viscosity but not the temperature.

${ }^{2}$ Recall that we assumed equal viscosities of the two fluids on either side of the plane of diffusion.
} 
because fluctuations can modify (renormalize) the Stokes-Einstein relationship [24, 30]. We return to this difference in Section $\mathrm{V}$, and for now we use a generic notation $\chi \approx \chi_{0}$ for the diffusion coefficient.

As explained in detail in [15, we start from (1) and define a concentration field from the positions of the particles via

$$
c(\boldsymbol{r}, t)=\sum_{i=1}^{N} \delta\left(\boldsymbol{q}_{i}(t)-\boldsymbol{r}\right) .
$$

For an ideal gas, Ito's rule formally gives a closed but nonlinear Ito stochastic advectiondiffusion equation for the concentration (c.f. Eq. (15) in [15]) ${ }^{3}$,

$$
\begin{aligned}
\partial_{t} c(\boldsymbol{r}, t) & =-\boldsymbol{\nabla} \cdot(\boldsymbol{w}(\boldsymbol{r}, t) c(\boldsymbol{r}, t))+\boldsymbol{\nabla} \cdot(\chi \nabla c(\boldsymbol{r}, t)) \\
& +\left(k_{B} T\right) \boldsymbol{\nabla} \cdot\left(c(\boldsymbol{r}, t) \int \boldsymbol{\mathcal { R }}\left(\boldsymbol{r}-\boldsymbol{r}^{\prime}\right) \boldsymbol{\nabla}^{\prime} c\left(\boldsymbol{r}^{\prime}, t\right) d \boldsymbol{r}^{\prime}\right) .
\end{aligned}
$$

Here $\boldsymbol{w}(\boldsymbol{r}, t)$ is a random velocity field that is white in time and has a spatial covariance,

$$
\left\langle\boldsymbol{w}(\boldsymbol{r}, t) \otimes \boldsymbol{w}\left(\boldsymbol{r}^{\prime}, t^{\prime}\right)\right\rangle=\left(2 k_{B} T\right) \boldsymbol{\mathcal { R }}\left(\boldsymbol{r}-\boldsymbol{r}^{\prime}\right) \delta\left(t-t^{\prime}\right)
$$

and has a clear physical interpretation in fluctuating hydrodynamics (FHD) as an overdamped representation of the fluid velocity [16].

The nonlinear term on the second line of (7) comes from the last term in (1) involving the divergence of the mobility matrix. Integrating by parts, we can rewrite the convolution as $-\left(k_{B} T\right) \boldsymbol{\nabla} \cdot\left(c(\boldsymbol{r}, t) \int\left(\boldsymbol{\nabla}^{\prime} \cdot \boldsymbol{\mathcal { R }}\left(\boldsymbol{r}-\boldsymbol{r}^{\prime}\right)\right) c\left(\boldsymbol{r}^{\prime}, t\right) d \boldsymbol{r}^{\prime}\right)$, which mimics the action of a pairwise force $\sim\left(k_{B} T\right) \boldsymbol{\nabla} \cdot \boldsymbol{\mathcal { R }}$. For the True3D or True2D Oseen and RPY tensors, the hydrodynamic kernel is divergence free, $\boldsymbol{\nabla} \cdot \boldsymbol{\mathcal { R }}(\boldsymbol{r})=0$, and so the nonlinear term in (7) disappears and we obtain a linear fluctuating advection-diffusion equation that can be solved numerically [16].

In the case of Quasi2D diffusion, however, the divergence of the mobility in the plane of confinement is nonzero and we must keep the second line of (7). In particular, in the far field (5) gives

$$
\boldsymbol{\nabla} \cdot \boldsymbol{\mathcal { R }}(\boldsymbol{r}) \approx \frac{1}{8 \pi \eta} \cdot \frac{\boldsymbol{r}}{r^{3}} \text { for } r \gg a
$$

\footnotetext{
${ }^{3}$ We have removed here a term $\boldsymbol{b}(\boldsymbol{r}, \boldsymbol{r}) c(\boldsymbol{r}, t)$ from Eq. (15) in [15] since this must vanish for translationallyinvariant systems, and deleted all terms coming from pairwise interactions.
} 
More precisely, in the equation for particle $i$ the last term in (1) looks like a Coulomb repulsion term ${ }^{4}$,

$$
\frac{d \boldsymbol{q}_{i}(t)}{d t} \approx \boldsymbol{w}\left(\boldsymbol{q}_{i}, t\right)+\sum_{j \neq i} \frac{k_{B} T}{8 \pi \eta} \cdot \frac{\boldsymbol{q}_{i}-\boldsymbol{q}_{j}}{\left\|\boldsymbol{q}_{i}-\boldsymbol{q}_{j}\right\|^{3}} .
$$

The last term in this equation has the same form as would have arisen in the absence of hydrodynamic interactions, $M_{i j}=\delta_{i j} /(6 \pi a \eta)$, had the particles been interacting with a repulsive "electrostatic" potential

$$
U_{\text {eff }}(r)=\frac{3 a}{4 r} k_{B} T
$$

Although this picture of the particles repelling each other with a long-ranged $r^{-1}$ potential is compelling and intuitive, it is also misleading because this "repulsion" is thermal in origin and comes from the rapid momentum transport perpendicular to the plane to which the particles are confined by a stiff potential. As such, the Quasi2D particle system is thermodynamically an ideal gas, and not a gas of point charges. The two terms on the right hand side of (9) are in fluctuation-dissipation balance with each other, and have a common origin in the thermal fluctuations of the fluid velocity.

As a nonlinear SPDE, (7) does not really make sense and it can be thought of simply as a rewriting of the equations of BD-HI; interestingly this kind of rewriting is particularly suited for constructing numerical methods to solve (11). In deriving (7), we have performed no coarse-graining other than forgetting the numbering of the particles, so the concentration is still a sum of delta functions [15]. However, for non-interacting particles, and for the case of interest to us where $\mathcal{R}$ is a long-ranged kernel, there is hope that (7) may nonetheless be useful in practice, after a suitable renormalization of the transport coefficients. The more traditional route [31], followed in [9], has been to write deterministic equations for the ensemble average by averaging over realizations of $\boldsymbol{w}$ (the same as averaging over the noise for the BD-HI equations). This, however, does not give a closed equation since one must then introduce the two-point correlation function, which is not known, as we discuss in great detail in Section IV. We therefore keep both the random advection and the nonlinear term in (7), as this is free of approximations and gives information not just about the ensemble average (which is hard to measure in experiments), but also about fluctuations present in each instance of the diffusive mixing process (which is what experiments measure). In this

\footnotetext{
${ }^{4}$ We have used here Eqs. (A7) and (A10) in [15, as we explain in more detail in Section III
} 
paper we study various nontrivial consequences of (7) and assess its usefulness by comparing results from DDFT-HI/FHD to results from BD-HI.

\section{Linearized Equations}

Let us first consider the case of a spatially uniform system with concentration $c(\boldsymbol{r}, t)=$ $c_{0}+\delta c(\boldsymbol{r}, t)$, where in some sense $\delta c \ll c_{0}$. Let us for a moment ignore the random advection, i.e., fluctuations, and simply consider the temporal relaxation of a small perturbation; the more complete derivation of the dynamic structure factor based on linearized FHD is given in Section VIB 1. If we linearize (7) around the uniform state we get the linear non-local diffusion equation

$$
\partial_{t} \delta c(\boldsymbol{r}, t)=\chi \boldsymbol{\nabla}^{2} \delta c(\boldsymbol{r}, t)+\left(k_{B} T\right) \boldsymbol{\nabla} \cdot\left(c_{0} \int \boldsymbol{\mathcal { R }}\left(\boldsymbol{r}-\boldsymbol{r}^{\prime}\right) \boldsymbol{\nabla}^{\prime} \delta c\left(\boldsymbol{r}^{\prime}, t\right) d \boldsymbol{r}^{\prime}\right) .
$$

This equation can trivially be solved in Fourier space to obtain

$$
\hat{\delta} c_{\boldsymbol{k}}(t)=\hat{\delta} c_{\boldsymbol{k}}(0) \exp \left(-k^{2} \chi_{c}(k) t\right)
$$

where hat denotes a Fourier transform ${ }^{5}$ and the subscript $\boldsymbol{k}$ denotes the wavenumber [32]. Here the short-time collective diffusion coefficient is

$$
\chi_{c}(k)=\chi+\left(k_{B} T\right) c_{0} k^{-2}\left(\boldsymbol{k} \cdot \hat{\boldsymbol{R}}_{\boldsymbol{k}} \cdot \boldsymbol{k}\right) .
$$

In this work we will not need to distinguish between short-time and long-time collective diffusion (see numerical results in Section VI A 1), and we therefore use $\chi_{c}$ to denote generically a collective diffusion coefficient.

Since $\boldsymbol{\mathcal { R }}$ and thus $\hat{\boldsymbol{R}}_{\boldsymbol{k}}$ are symmetric positive semidefinite kernels, $\boldsymbol{k} \cdot \hat{\boldsymbol{\mathcal { R }}}_{\boldsymbol{k}} \cdot \boldsymbol{k} \geq 0$ and in general as the density $c_{0}$ increases the collective diffusion is potentially accelerated over the case without hydrodynamics, $\chi_{c} \equiv \chi$. This somewhat unexpected collective effect does not exist in either True2D or True3D because of the incompressibility of the fluid flow, $\boldsymbol{k} \cdot \hat{\mathcal{R}}_{\boldsymbol{k}} \cdot \boldsymbol{k}=0$. The collective enhancement of $\chi_{c}$ becomes important for Quasi2D diffusion and has been studied in a number of prior works [8, 10, 14].

\footnotetext{
${ }^{5}$ The scaling convention for the Fourier transforms that we use is given in [32, and ensures that the Fourier spectrum of white noise is unity.
} 
Note that our derivation exposes that the only approximation made for an ideal gas of particles is the linearization. Although this linearization seems natural, we will see in Section $\mathrm{V}$ that even for an ideal gas this is only an approximation, and that fluctuations renormalize the diffusion coefficient $\chi$ by a measurable amount through a nonlinear (mode) coupling. The derivation in [9] is in our opinion unnecessarily complicated as it invokes unnecessary closures to derive the same result (13). This is because the authors of [9] start from the DDFT-HI equations for the ensemble average [31], which are not closed, so they need a number of approximations to arrive at the same equation. Of course, for interacting particles one must invoke closures, perhaps in the form of density functionals [31, and make additional approximations that are very difficult to justify mathematically.

\section{Oseen Approximation in Quasi2D}

To leading order in the far field, i.e., for small $k a \ll 1$, we can compute $\hat{\mathcal{R}}_{\boldsymbol{k}}$ in Quasi2D by simply integrating the well-known Fourier transform of the 3D Oseen tensor along the $z$ axes,

$$
\hat{\boldsymbol{R}}_{\boldsymbol{k}}=\frac{1}{2 \pi \eta} \int_{k_{z}=-\infty}^{\infty} \frac{d k_{z}}{\left(k^{\prime}\right)^{2}}\left(\boldsymbol{I}-\frac{\boldsymbol{k}^{\prime} \otimes \boldsymbol{k}^{\prime}}{\left(k^{\prime}\right)^{2}}\right),
$$

where $\boldsymbol{k}=\left(k_{x}, k_{y}\right)$ and $\boldsymbol{k}^{\prime}=\left(k_{x}, k_{y}, k_{z}\right)$. The integral can be done analytically [8 10], to give the spectral decomposition

$$
\hat{\mathcal{R}}_{\boldsymbol{k}}=\frac{1}{\eta k^{3}}\left(\frac{1}{2} \boldsymbol{k}_{\perp} \otimes \boldsymbol{k}_{\perp}+\frac{1}{4} \boldsymbol{k} \otimes \boldsymbol{k}\right),
$$

where $\boldsymbol{k}_{\perp}=\left(k_{y},-k_{x}\right)$ is a vector perpendicular to $\boldsymbol{k}$, i.e., $\boldsymbol{k}_{\perp}=\boldsymbol{k} \times \hat{z}$.

This shows that the spectrum of the random velocity field $\boldsymbol{w}(\boldsymbol{r}, t)$ decays like $1 / k$ and that the field is compressible. Specifically, for the divergence we get $\boldsymbol{k} \cdot \hat{\boldsymbol{R}}_{\boldsymbol{k}} \cdot \boldsymbol{k}=k / 4 \eta$, which allows us to write 13 in the form

$$
\chi_{c}(k)=\chi\left(1+\frac{1}{k L_{h}}\right)
$$

where $L_{h}=4 \eta \chi /\left(k_{B} T c_{0}\right)=2 /\left(3 \pi a c_{0}\right)$ is a hydrodynamic correlation length. Remarkably, for $\mathrm{q} 2 \mathrm{D}$ diffusion $\chi_{c}$ diverges like $1 / k[8-10]$. For planar packing densities $\phi=\pi c_{0} a^{2} \sim 1$, which is the most interesting regime for collective diffusion, we have $L_{h} \sim a$ and therefore collective diffusion effects manifest themselves strongly at all length scales of interest. 
Note that the real space equivalent of (15) shows a power law $1 / r^{3}$ tail rather than a Gaussian tail as it does for ordinary diffusion [8, 9]. This is yet another dramatic consequence of the nonzero divergence of the flow in the plane of confinement that we explore further in Section IV.

\section{BROWNIAN DYNAMICS IN QUASI TWO-DIMENSIONS}

In this section we develop a novel algorithm to perform BD-HI in (quasi-)two-dimensional systems (BD-2D) in linear time in the number of particles. This improves dramatically the efficiency over prior methods for strict confinement [10] or partial confinement [13, 14]. The algorithm is based on the DDFT-HI equation (7), viewed from the perspective of fluctuating hydrodynamics [16]. A closely-related numerical method implemented in the GPU code fluam [33, 34] has previously been used by some of us with partial confinement [13]. The key new step here is to assume perfect confinement and eliminate completely the third dimension, thus significantly reducing the computational complexity at the expense of introducing a nonzero divergence of the flow. Our algorithm combines the Fluctuating Force Coupling Method (F-FCM) [35, 36] with ideas used in the Fluctuating Immersed Boundary method (FIB) 34 to construct a simple yet efficient algorithm specifically tailored to periodic twodimensional systems, with either True2D and Quasi2D hydrodynamics.

A rather general form of the hydrodynamic kernel is given by the double convolution

$$
\mathcal{R}\left(\boldsymbol{r}_{1}, \boldsymbol{r}_{2}\right)=\mathcal{R}\left(\boldsymbol{r}_{1}-\boldsymbol{r}_{2}\right)=\int \delta_{a}\left(\boldsymbol{r}_{1}-\boldsymbol{r}^{\prime}\right) \mathbb{G}\left(\boldsymbol{r}^{\prime}-\boldsymbol{r}^{\prime \prime}\right) \delta_{a}\left(\boldsymbol{r}_{2}-\boldsymbol{r}^{\prime \prime}\right) d \boldsymbol{r}^{\prime} d \boldsymbol{r}^{\prime \prime}
$$

where $\mathbb{G}$ is a Green's function for the particular kind of flow (True2D, Quasi2D, or True3D, or other), and $\delta_{a}$ are regularizing kernels (i.e., smeared delta functions) of width $a$. The form (17) is consistent consistent with both the F-FCM and FIB methods, as well as recent BD-HI algorithms based on the RPY kernel [37]. For RPY, the delta kernels are surface delta functions on the surface of a sphere of radius $a$, and for FCM the delta kernels are Gaussian kernels with standard deviation $\sigma=a / \sqrt{\pi}$ [38]. It is important to note that for Quasi2D the kernels and the intergrals in (17) are three dimensional even though the target $\boldsymbol{r}_{1}$ and source $\boldsymbol{r}_{2}$ lie in the plane of the interface. By contrast, for True2D diffusion there is no third dimension and the kernels and intergrals in (17) are both two dimensional.

In this work we employ Gaussian kernels for $\delta_{a}$ as in the FCM because this: (1) allows for 
spectral accuracy of the numerical method, without the near-field corrections required for the RPY kernel [37]; (2) avoids small-scale grid artifacts present for compactly-supported kernels as used in the FIB method; and (3) simplifies analytical computations. At the level of accuracy of the Rotne-Prager pairwise approximation, there is no strong reason to prefer the RPY kernel over the FCM kernel, as they both capture the far-field hydrodynamics to a similar accuracy [38, and regularize the near-field hydrodynamics in a similar manner.

Using the translational invariance and isotropy of (17) to simplify Eqs. (A7) and (A10) in [15, and using (17), and it is not hard to show that the BD-HI equations (1) can be written in the form

$$
\frac{d \boldsymbol{q}_{i}}{d t}=\boldsymbol{w}\left(\boldsymbol{q}_{i}, t\right)+\int \delta_{a}\left(\boldsymbol{q}_{i}-\boldsymbol{r}^{\prime}\right) \sum_{j} \mathbb{G}\left(\boldsymbol{r}^{\prime}, \boldsymbol{r}^{\prime \prime}\right)\left[\boldsymbol{F}_{j} \delta_{a}\left(\boldsymbol{q}_{j}-\boldsymbol{r}^{\prime \prime}\right)+\left(k_{B} T\right)\left(\boldsymbol{\partial} \delta_{a}\right)\left(\boldsymbol{q}_{j}-\boldsymbol{r}^{\prime \prime}\right)\right] d \boldsymbol{r}^{\prime} d \boldsymbol{r}^{\prime \prime}
$$

Here $\partial_{\alpha} \delta_{a}(\boldsymbol{r})=\partial \delta_{a}(\boldsymbol{r}) / \partial r_{\alpha}$ denotes the gradient of the Gaussian kernel $\delta_{a}(\boldsymbol{r})$. It is important to emphasize that this gradient is taken in the ambient space in which the diffusion happens, i.e., in the plane of confinement for the case of True2D and Quasi2D diffusion, and in three-dimensional space for True3D diffusion. Here we have made the sum include $i=j$ because the self term disappears by the fact the kernel and its gradient are rotationally isotropic. Note that one can perform an integration by parts and move the gradient from $\delta_{a}$ to the Green's function as a divergence to show that the last term can be omitted when the Green's function is divergence free in the space in which the diffusion happens (e.g., for True2D and True3D).

The particles-only equation (18) is the overdamped and incompressible limit of coupled fluctuating fluid-particle equations that include inertia 33. We can gain physical intuition about the term $\left(k_{B} T\right)\left(\boldsymbol{\partial} \delta_{a}\right)\left(\boldsymbol{q}_{j}-\boldsymbol{r}^{\prime \prime}\right)$ in 18$)$, which is the cause of the collective diffusion enhancement, by observing that it arises due to the osmotic pressure associated with the colloidal particle itself [39]. This osmotic pressure contribution has been justified from a coarse-graining perspective in [40].

\section{A. Force Coupling Method in Quasi2D}

One can solve (9) very efficiently pseudo-spectrally [16] once an explicit form for the Fourier transform of the hydrodynamic kernel is available. This Fourier transform must 
have the isotropic form

$$
\hat{\boldsymbol{R}}_{\boldsymbol{k}}=\frac{1}{\eta k^{3}}\left(c_{2}(k a) \boldsymbol{k}_{\perp} \otimes \boldsymbol{k}_{\perp}+c_{1}(k a) \boldsymbol{k} \otimes \boldsymbol{k}\right)
$$

where we singled out a $1 / k^{3}$ prefactor mimicking (15). The Fourier transform of the Quasi2D FCM kernel can easily be computed by multiplying the integrand in (14) by the square of the Fourier transform of the Gaussian kernel in agreement with (17),

$$
\hat{\boldsymbol{R}}_{\boldsymbol{k}}=\frac{1}{2 \pi \eta} \int_{k_{z}=-\infty}^{\infty} \frac{d k_{z}}{\left(k^{2}+k_{z}^{2}\right)} \exp \left(-\frac{a^{2}\left(k^{2}+k_{z}^{2}\right)}{\pi}\right)\left(\boldsymbol{I}-\frac{\left(\boldsymbol{k}, k_{z}\right) \otimes\left(\boldsymbol{k}, k_{z}\right)}{\left(k^{2}+k_{z}^{2}\right)}\right) .
$$

Performing the integrals gives

$$
\begin{aligned}
& c_{1}(K)=\frac{1}{2 \pi}\left(-K \exp \left(-\frac{K^{2}}{\pi}\right)-\left(K^{2}+\frac{\pi}{2}\right)\left(\operatorname{erf}\left(\frac{K}{\sqrt{\pi}}\right)-1\right)\right) \\
& c_{2}(K)=\frac{1}{2}\left(1-\operatorname{erf}\left(\frac{K}{\sqrt{\pi}}\right)\right)
\end{aligned}
$$

in Quasi2D. An important point is that both expression decay exponentially like $\exp \left(-a^{2} k^{2}\right)$ in Fourier space, which is crucial for pseudospectral methods to obtain spectral accuracy. For an unbounded domain, the FCM correlation tensor $\mathcal{R}(\boldsymbol{r})$ can be computed in real space from the expressions given in [38] and has the form (4) with the functions

$$
\begin{aligned}
& f(r)=\frac{1}{8 \pi \eta r}\left(\left(1+2 \frac{a^{2}}{\pi r^{2}}\right) \operatorname{erf}\left(\frac{r \sqrt{\pi}}{2 a}\right)-2 \frac{a}{\pi r} \exp \left(-\frac{\pi r^{2}}{4 a^{2}}\right)\right), \\
& g(r)=\frac{1}{8 \pi \eta r}\left(\left(1-6 \frac{a^{2}}{\pi r^{2}}\right) \operatorname{erf}\left(\frac{r \sqrt{\pi}}{2 a}\right)+6 \frac{a}{\pi r} \exp \left(-\frac{\pi r^{2}}{4 a^{2}}\right)\right) .
\end{aligned}
$$

In agreement with 15$]$, for small wavenumbers we have

$$
\begin{aligned}
& c_{1}(K=k a \ll 1) \approx \frac{1}{4} \\
& c_{2}(K=k a \ll 1) \approx \frac{1}{2} .
\end{aligned}
$$

We can unify True2D and Quasi2D by defining

$$
\begin{aligned}
c_{1}(K) & =0 \\
c_{2}(K) & =\frac{a}{K} \exp \left(-\frac{K^{2}}{\pi}\right),
\end{aligned}
$$

for True2D. We have chosen to keep the standard deviation of the Gaussian kernel $\delta_{a}$ the same as in True3D, since this empirically reproduces the diffusion coefficient of a no-slip 
disk of radius $a$, see 23$)$. Note that in True2D we have $c_{2}(k a \ll 1) \approx 1 / k$. We can also generically write the short-time self-diffusion coefficient as

$$
\chi_{0}=\left(k_{B} T\right) f(r=0)=\beta \frac{k_{B} T}{\eta}
$$

where the factor $\beta$ is 30 .

$$
\begin{aligned}
& \beta=\frac{1}{6 \pi a} \cdot \frac{1}{1+4.41 a / L} \approx \frac{1}{6 \pi a} \text { for Quasi2D, and } \\
& \beta=\frac{1}{4 \pi} \ln \left(\frac{L}{3.71 a}\right) \quad \text { for True2D, }
\end{aligned}
$$

and $L$ is the size of the square periodic unit cell. We have included for Quasi2D the leadingorder finite-size correction, where we estimated the coefficient 4.41 numerically. Observe that in True2D the diffusion coefficient diverges logarithmically with system size, and the empirical coefficient 3.71 matches that for a no-slip disk of radius $a$ [41, although we have not been able to confirm this analytically.

From 19 we get for the flow divergence

$$
c_{0}\left(k_{B} T\right) \boldsymbol{k} \cdot \hat{\boldsymbol{R}}_{\boldsymbol{k}} \cdot \boldsymbol{k}=c_{0}\left(\frac{k_{B} T}{\eta}\right) k c_{1}(a k)=6 \pi \chi a c_{0} k c_{1}(a k)=\left(\chi k^{2}\right) \frac{4 c_{1}(k a)}{k L_{h}},
$$

giving the collective diffusion coefficient

$$
\chi_{c}(k)=\chi\left(1+\frac{4 c_{1}(k a)}{k L_{h}}\right) .
$$

This formula extends (16) to all wavenumbers for the FCM kernel.

\section{B. Efficient Two-Dimensional Brownian Dynamics}

Equation (18) is the basis of our efficient BD-2D algorithm, summarized in Algorithm 1. We now explain the key ideas behind the steps of the algorithm. This algorithm is now part of the public-domain GPU code fluam, available freely at https://github.com/ fbusabiaga/fluam.

The second term in (18) can be captured by first spreading to the grid the force density $\boldsymbol{F}_{j} \delta_{a}+\left(k_{B} T\right) \boldsymbol{\partial} \delta_{a}$ localized around each particle $j$, and then solving the fluid equations in Fourier space by performing the convolution with $\mathbb{G}$ in $(18)$ using FFTs. Convolution by $\mathbb{G}$

amounts to multiplication by $\hat{\mathbb{G}}_{\boldsymbol{k}}$ in Fourier space. In Fourier space, the FCM representation 
$\mathbb{G}$ is given by 19 but now without the Gaussian "delta" functions in $x$ and $y$ since these are added by the explicit spatial convolution in (18). Specifically, in Quasi2D

$$
\begin{aligned}
\hat{\mathbb{G}}_{\boldsymbol{k}} & =\frac{1}{2 \pi \eta} \int_{k_{z}=-\infty}^{\infty} \frac{d k_{z}}{\left(k^{2}+k_{z}^{2}\right)} \exp \left(-\frac{a^{2} k_{z}^{2}}{\pi}\right)\left(\boldsymbol{I}-\frac{\left(\boldsymbol{k}, k_{z}\right) \otimes\left(\boldsymbol{k}, k_{z}\right)}{\left(k^{2}+k_{z}^{2}\right)}\right)= \\
& =\hat{\mathcal{R}}_{\boldsymbol{k}} \exp \left(\frac{a^{2} k^{2}}{\pi}\right)=\frac{1}{\eta}\left[g_{k}(k a) \boldsymbol{k}_{\perp} \otimes \boldsymbol{k}_{\perp}+f_{k}(k a) \boldsymbol{k} \otimes \boldsymbol{k}\right],
\end{aligned}
$$

where the explicit form of $f_{k}$ and $g_{k}$ can be read from (19). It is important to observe that the convolution by a Gaussian in the $z$ direction is included in the integral above since our FFTs and grid are purely two dimensional. In True2D, the Green's function is the familiar Green's function for 2D Stokes flow, given by $f_{k}=0$ and $g_{k}=1 /\left(\eta k^{2}\right)$.

The first term in (18) represents advection by a random velocity. This velocity can be generated in Fourier space using (26). From (19) we get the Fourier representation required to generate the random velocity efficiently,

$$
\widehat{\boldsymbol{w}}_{\boldsymbol{k}}=\sqrt{\frac{2 k_{B} T}{\eta k^{3}}}\left(\sqrt{c_{2}(k a)} \boldsymbol{k}_{\perp} \mathcal{Z}_{\boldsymbol{k}}^{(2)}+\sqrt{c_{1}(k a)} \boldsymbol{k} \mathcal{Z}_{\boldsymbol{k}}^{(1)}\right)
$$

where $\mathcal{Z}_{\boldsymbol{k}}^{(1 / 2)}(t)$ are scalar white noise processes, independent of each other and independent between different wavenumbers. The random Gaussian variables $\mathcal{Z}_{k}^{(1 / 2)}$ should obey the symmetry properties required to ensure that the velocity field is real-valued in real space; see detailed discussion in Section 3.3 in [42. Note that the same formula $(26)$ works for True2D and Quasi2D using the appropriate definitions of $c_{1}$ and $c_{2}$. In our algorithm the Gaussian factor $\exp \left(-a^{2} k^{2} / \pi\right)$ is included when interpolating the random velocity at the particle positions, so in (27) in the algorithm the random velocity is generated using the factors $f_{k} / g_{k}$ and not the factors $c_{1} / c_{2}$.

The grid used to perform the FFTs in Algorithm 1 must be sufficiently fine to accurately resolve the convolutions with the Gaussian kernel $\exp \left(-a^{2} k^{2} / \pi\right)$ and to capture all of the active modes in the fluid velocity. We have numerically estimated that the grid spacing $h$ should satisfy $h \lesssim 0.8 a$ to ensure a relative error smaller than $10^{-4}$ in the short-time self diffusion coefficient; the precise value of $h$ should be chosen to ensure that the number of grid cells factorizes favorably to speed up the FFT. The Gaussian kernels need to be cut off in real space after a distance $r_{c}$ to ensure that the kernel $\delta_{a}$ is compactly supported [43]. We have numerically estimated that $r_{c} \geq 2.5 a$ is appropriate for a relative error tolerance of $10^{-4}$. This cutoff can be efficiently implemented by using a mask of $(2 P+1) \times(2 P+1)$ cells 
$\overline{\text { Algorithm } 1 \text { Outline of the } n \text {-th time step in an efficient algorithm for performing BD-HI }}$ in two dimensions (BD-2D). The time step size is denoted with $\Delta t$. Although we employ continuum notation for simplicity, the actual implementation uses a grid to discretize the

fields, and uses the trapezoidal rule to discretize the spatial integrals. This algorithm works for any translationally invariant isotropic hydrodynamic interaction kernel.

1. Evaluate particle forces $\boldsymbol{F}^{n}=\boldsymbol{F}\left(\boldsymbol{Q}^{n}\right)$.

2. Compute in real space on a grid the fluid forcing

$$
\boldsymbol{f}(\boldsymbol{r})=\sum_{i} \boldsymbol{F}_{i} \delta_{a}\left(\boldsymbol{q}_{i}-\boldsymbol{r}\right)+\left(k_{B} T\right) \sum_{i}\left(\boldsymbol{\partial} \delta_{a}\right)\left(\boldsymbol{q}_{i}-\boldsymbol{r}\right)+\boldsymbol{f}_{\mathrm{ext}}(\boldsymbol{r}),
$$

and use the FFT to convert $f$ to Fourier space, i.e., to compute $\hat{f}_{k}$. For True2D we omit the second term since the hydrodynamic tensor is divergence free. Here $\boldsymbol{f}_{\text {ext }}(\boldsymbol{r})$ is an external force on the fluid (e.g., gravity), which can be used to perform non-equilibrium measurements.

3. Compute the fluid velocity resulting from the fluid forcing $f$ in Fourier space as a convolution with the Green's function (25),

$$
\hat{\boldsymbol{v}}_{k}^{\operatorname{det}}=\hat{\mathbb{G}}_{k} \hat{f}_{k} .
$$

4. Generate a random fluid velocity with covariance $\left(2 k_{B} T / \Delta t\right) \hat{\mathbb{G}}_{k}$ in Fourier space,

$$
\hat{\boldsymbol{v}}_{k}^{\text {stoch }}=\sqrt{\frac{2 k_{B} T}{\eta \Delta t}}\left(\sqrt{g_{k}(k)} \boldsymbol{k}_{\perp} W_{\boldsymbol{k}}^{(2)}+\sqrt{f_{k}(k)} \boldsymbol{k} W_{\boldsymbol{k}}^{(1)}\right),
$$

where $W_{k}^{(1 / 2)}$ are standard random Gaussian variables with the symmetry properties required to make $\boldsymbol{v}^{\text {stoch }}(\boldsymbol{r})$ real valued.

5. Compute the total fluid velocity

$$
\hat{\boldsymbol{v}}_{k}=\hat{\boldsymbol{v}}_{k}^{\mathrm{det}}+\hat{\boldsymbol{v}}_{k}^{\mathrm{stoch}}
$$

and use the FFT to convert it to real space and obtain $\boldsymbol{v}(\boldsymbol{r})$.

6. Convolve $\boldsymbol{v}(\boldsymbol{r})$ with a Gaussian in real space to compute particle velocities,

$$
\boldsymbol{u}_{i}=\int \delta_{a}\left(\boldsymbol{q}_{i}-\boldsymbol{r}\right) \boldsymbol{v}(\boldsymbol{r}) d \boldsymbol{r}
$$

7. Advance the particles' positions,

$$
\boldsymbol{q}_{i}^{n+1}=\boldsymbol{q}_{i}^{n}+\boldsymbol{u}_{i} \Delta t
$$


when spreading the forces to the grid and interpolating the velocity from the grid, where $P=4$ for $h=0.8 a$.

The temporal integration scheme in Algorithm 1 is similar to that used in the FIB method [34]; see in particular the simple midpoint scheme presented in section IV.A of [34]. We have, however, made several simplifications using the fact that to high numerical accuracy the hydrodynamic kernel is translationally-invariant, as well as the fact that we can analytically differentiate Gaussian kernels to compute $\boldsymbol{\partial} \delta_{a}$ without requiring a so-called random finite difference [34]. A delicate point is choosing the appropriate time step size $\Delta t$. A demanding test that can be used to assess the magnitude of the temporal integration error is to examine the equilibrium pair correlation function $g_{2}(r)$, or, equivalently, the equilibrium structure factor $S(k)$. For interacting particles the time step size is typically limited by the stiffness of the steric repulsion; however, even for non-interacting particles (ideal gas) the time step must be small enough to ensure that particles move only a fraction of their hydrodynamic radius in a time step, $\chi \Delta t \ll a^{2}$. This is the only requirement for an ideal gas in True2D, however, in Quasi2D the particles "interact" through the thermal fluctuations they induce in the fluid via their osmotic pressure. We empirically observe that in Quasi2D, the higher the density, and thus the stronger the collective effects, the smaller the time step must be in order to maintain the accuracy of $g_{2}(r)$. The largest error in $g_{2}$ was found to be for $r \rightarrow 0^{+}$.

Most of the simulations reported in this work employ one of two setups, Setup A for planar packing density $\phi=0.5$ or Setup B for $\phi=1$. The relevant parameters are given in Table I.

\section{Linear response theory}

One of the key properties of the dynamics (1) is that it is in detailed balance (i.e., it is time reversible) with respect to an ideal gas equilibrium distribution. The thermodynamic equilibrium state of the suspension is consistent with that of an ideal gas. We have verified that our numerical method satisfies this property by confirming that the pair correlation function $g_{2}(r)=1$ for sufficiently small time step sizes (not shown). In order to confirm that our algorithm preserves the time-reversibility of the dynamics we now test a key prediction of linear response theory (LRT) [44], which is based on time reversibility at equilibrium. 


\begin{tabular}{|c|c|c|c|c|c|}
\hline Setup & $\phi$ & $L$ & $N$ & $k_{B} T, \eta$ and $a$ & $N_{\mathrm{FFT}}^{2}$ \\
\hline \hline A & 0.5 & 800 & $10^{5}$ & 1 & $1152^{2}$ \\
\hline B & 1.0 & 560.5 & $10^{5}$ & 1 & $864^{2}$ \\
\hline
\end{tabular}

Table I: Parameters used for the majority of the simulations reported in this work, unless otherwise indicated, in arbitrary units. All simulations use a periodic domain of length $L_{x}=L_{y}=L$ with a total of $N$ particles covered by an FFT grid of size $N_{\text {FFT }}^{2}$ cells. In Setup A, we set $L=800$ giving a packing density (fraction) $\phi=c_{0} \pi a^{2} \approx 0.5$, where $c_{0}=N /\left(L_{x} L_{y}\right)$ is the equilibrium number density (concentration). In Setup B, we set $L=560.5$ to get a higher packing density $\phi=1.0$ and thus stronger collective effects. Unless otherwise noted, in Quasi2D, we use a time step size $\Delta t=0.2$, and in True2D we use $\Delta t=0.05$ because the diffusion coefficient is larger; these values were chosen to ensure an error less than $10 \%$ in $g_{2}\left(r \rightarrow 0^{+}\right)$at equilibrium, even for the larger density in Setup B.

Specifically, we analyze the mean square displacement (MSD) of a tagged particle $p$,

$$
\operatorname{MSD}(t)=\operatorname{MSD}_{x}(t)+\operatorname{MSD}_{y}(t)=\left\langle\left(x_{p}(t)-x_{p}(0)\right)^{2}\right\rangle+\left\langle\left(y_{p}(t)-y_{p}(0)\right)^{2}\right\rangle .
$$

This can be computed at equilibrium by averaging the square displacements over all of the particles. It can also be computed using a non-equilibrium method based on LRT. If we pull a tagged particle $p$ with a force $\boldsymbol{F}=F \hat{\boldsymbol{x}}$, LRT predicts that

$$
\left\langle x_{p}(t)-x_{p}(0)\right\rangle_{\boldsymbol{F}}=-\frac{F}{k_{B} T} \int_{0}^{t}\left\langle x_{p}(0) \dot{x}_{p}\left(t-t^{\prime}\right)\right\rangle_{0} \mathrm{~d} t^{\prime}=\frac{F}{2 k_{B} T}\left\langle\left(x_{p}(t)-x_{p}(0)\right)^{2}\right\rangle_{0} .
$$

The average on the left hand side is an average over nonequilibrum trajectories initialized from the equilibrium distribution, while the average on the right hand side is an average over equilibrium trajectories in the absence of the forcing. The formula (28) relates the MSD at equilibrium with the mean displacement under a external force. In the left panel of Fig. 1 we confirm that this relation is maintained by our Quasi2D BD-HI (BD-q2D for short) algorithm, confirming that the algorithm preserves detailed balance (time reversibility).

More interestingly, we observe that the MSD is not strictly linear in time. While the shorttime slope of the MSD is consistent with the short-time self-diffusion coefficient $\chi_{s}^{(s)}=\chi_{0}$ predicted by the Stokes-Einstein relation (22), the long-time slope gives a smaller long-time 


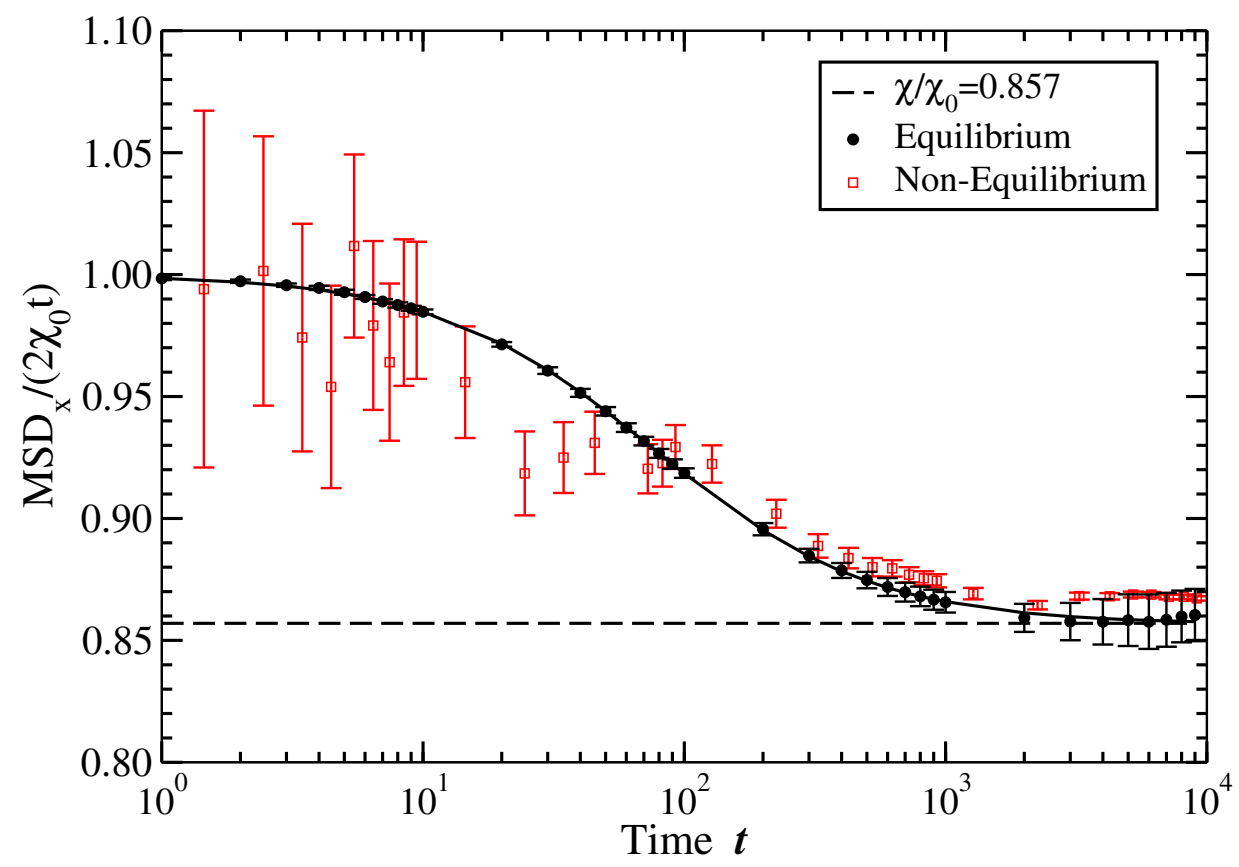

Figure 1: Mean square displacement of a tagged particle in a Quasi2D ideal gas of density $\phi=1$, computed from the equilibrium MSD (filled black circles), as well as the nonequilibrium average displacement of a particle pulled by a force $F=1$ (empty squares). Error bars indicate one standard deviation confidence intervals. Note that the statistical errors are quite large for the nonequilibrium measurements, especially at short times, because only a single particle is pulled per simulation, whereas the MSD is averaged over all particles. Parameters are based on Setup B (see Table I except that $\Delta t=0.1$ to better capture the short time behavior and reduce the error in $g_{2}(r)$.

self-diffusion coefficient $\chi_{s}^{(l)} \approx 0.85 \chi_{0}$ (for this packing density $\left.\phi=1\right)^{6}$. We return to this unexpected effect in greater detail in Section $\mathrm{V}$.

\section{DYNAMICS OF THE ENSEMBLE AVERAGE}

In this section we study the evolution of the ensemble average or mean of the total density [9] and the density of a labeled species (tracer) in Quasi2D diffusion. While for

${ }^{6}$ It is worth noting that the equality $\chi_{s}^{(s)}(\phi)=\chi_{0}=\lim _{\phi \rightarrow 0} \chi_{s}^{(l)}(\phi)$ is built into the Rotne-Prager or pairwise approximation of the mobility we use in this work. When realistic near-field hydrodynamics is accounted for, $\chi_{s}^{(s)}(\phi)$ will in general be different from $\chi_{0}$ for nonvanishing densities. 
True2D hydrodynamics the mean strictly follows the familiar Fick law (diffusion equation), as it would in the absence of hydrodynamics (i.e., for uncorrelated random walkers), this does not carry on to Quasi2D systems.

\section{A. Ensemble average for density}

As a nonlinear SPDE, (7) does not really make sense mathematically, and it can be thought of simply as a rewriting of (1). To obtain an ensemble average we need to average over realizations of $\boldsymbol{w}$ (i.e., over stochastic realizations), but this does not give a closed equation and we must also introduce the unknown two-point correlation function $c^{(2)}\left(\boldsymbol{r}, \boldsymbol{r}^{\prime}, t\right)$. Specifically, ensemble averaging (7) gives [15, 31]

$$
\partial_{t} c^{(1)}(\boldsymbol{r}, t)=\boldsymbol{\nabla} \cdot\left(\boldsymbol{\chi}(\boldsymbol{r}) \boldsymbol{\nabla} c^{(1)}(\boldsymbol{r}, t)\right)+\left(k_{B} T\right) \boldsymbol{\nabla} \cdot\left(\int \boldsymbol{\mathcal { R }}\left(\boldsymbol{r}, \boldsymbol{r}^{\prime}\right) \boldsymbol{\nabla}^{\prime} c^{(2)}\left(\boldsymbol{r}, \boldsymbol{r}^{\prime}, t\right) d \boldsymbol{r}^{\prime}\right)
$$

where the single-particle distribution function (mean number density) is $c^{(1)}(\boldsymbol{r}, t)=\langle c(\boldsymbol{r}, t)\rangle$. For an ideal gas, the standard closure for the two-particle correlation function is [31, 45]

$$
c^{(2)}\left(\boldsymbol{r}, \boldsymbol{r}^{\prime}, t\right) \approx c^{(1)}(\boldsymbol{r}, t) c^{(1)}\left(\boldsymbol{r}^{\prime}, t\right) .
$$

After making this approximation in (29) we get the closed nonlinear nonlocal diffusion equation

$$
\partial_{t} c^{(1)}(\boldsymbol{r}, t)=\nabla \cdot\left(\chi(\boldsymbol{r}) \boldsymbol{\nabla} c^{(1)}(\boldsymbol{r}, t)+\left(k_{B} T\right) c^{(1)}(\boldsymbol{r}, t) \int \mathcal{R}\left(\boldsymbol{r}, \boldsymbol{r}^{\prime}\right) \boldsymbol{\nabla}^{\prime} c^{(1)}\left(\boldsymbol{r}^{\prime}, t\right) d \boldsymbol{r}^{\prime}\right),
$$

which can be solved efficiently and accurately using standard pseudospectral methods. Note that this equation looks exactly like the fluctuating (7) but without the noise term. This suggests that this approximation can also be thought of as neglecting fluctuations. It is important to note that in True2D or True3D (30) reduces to the standard (linear) diffusion equation $\partial_{t} c^{(1)}=\chi \nabla^{2} c^{(1)}$ after integration by parts, and is an exact consequence of (1); divergence-free hydrodynamic interactions affect the spectrum of the fluctuations but do not alter the mean [16. In Quasi2D, however, (30) is only an approximation (i.e., a closure) that must be tested numerically.

We compare results from BD-HI simulations with a numerical solution of (30) in the left panel of Fig. 2. In this numerical experiment, we use parameters from Setup A (see Table I), $\phi=0.5$, and follow the dynamics up to time $T=2100$. We average the numerical results over 
one thousand simulations. In the initial configuration, we randomly and uniformly distribute $0.9 \cdot 10^{5}$ particles throughout the domain, and then randomly and uniformly distribute an additional $0.1 \cdot 10^{5}$ particles in the stripe $-L_{y} / 6 \leq y \leq L_{y} / 6$. This creates a one-dimensional density gradient along the $y$ direction, see black dashed line in the left panel of Fig. 2; see Fig. 8 for a visual representation in a similar setup. In what follows we study the density profile averaged along the $x$ axis, i.e., we study the ensemble average $c^{(1)}(y, t)=\langle c(y, t)\rangle$ of $c(y, t)=\int_{x=0}^{L_{x}} c(x, y, t) d x$.

In the left panel of Fig. 2 we compare results for $c^{(1)}(y, T)$ for a system without hydrodynamic interactions (black solid line) and a system with Quasi2D hydrodynamics (red squares, BD-q2D) to the numerical solution of (30) in Quasi2D (red solid line). Note that the case without hydrodynamics is exactly described by the standard diffusion equation. The good agreement between the numerical results for BD-q2D and the theoretical predictions suggests that the mean-field approximation $(30)$ is reasonable. When solving (30) we

used $\chi=\chi_{s}^{(s)}=\chi_{0}$ and not the long-time self diffusion coefficient $\chi_{s}^{(l)} \approx 0.88 \chi_{0}$ (at this density $\phi=0.5$, see Fig. 1); however, the difference between the two values is too small to appreciate on the scales of Fig. 2 because the nonlinear term in (30) dominates.

\section{B. Ensemble average for color}

As already studied in detail in prior work [8, 9], the evolution of density perturbations in Quasi2D is very different from the familiar diffusive decay, not just in the time domain but also in the space domain. In particular, density perturbations spread much further in Quasi2D and develop inverse cubed power-law tails instead of the familiar Gaussian tails. Such an algebraic spatial decay of localized density perturbations is quite unexpected and unusual, and raises some questions. Do some/all/few particles quickly displace very far, so as to create a power-law tail for $t>0$ ? Or, do all particles still displace by small diffusive displacements, but a power-law tail arises in the average due to the inter-particle correlations?

To further interrogate the individual particle displacements we color (label) the particles "red" and "green". This could be done in experiments by using fluorescent labels. The generalization of (30) to account for species labels is straightforward to write down and 

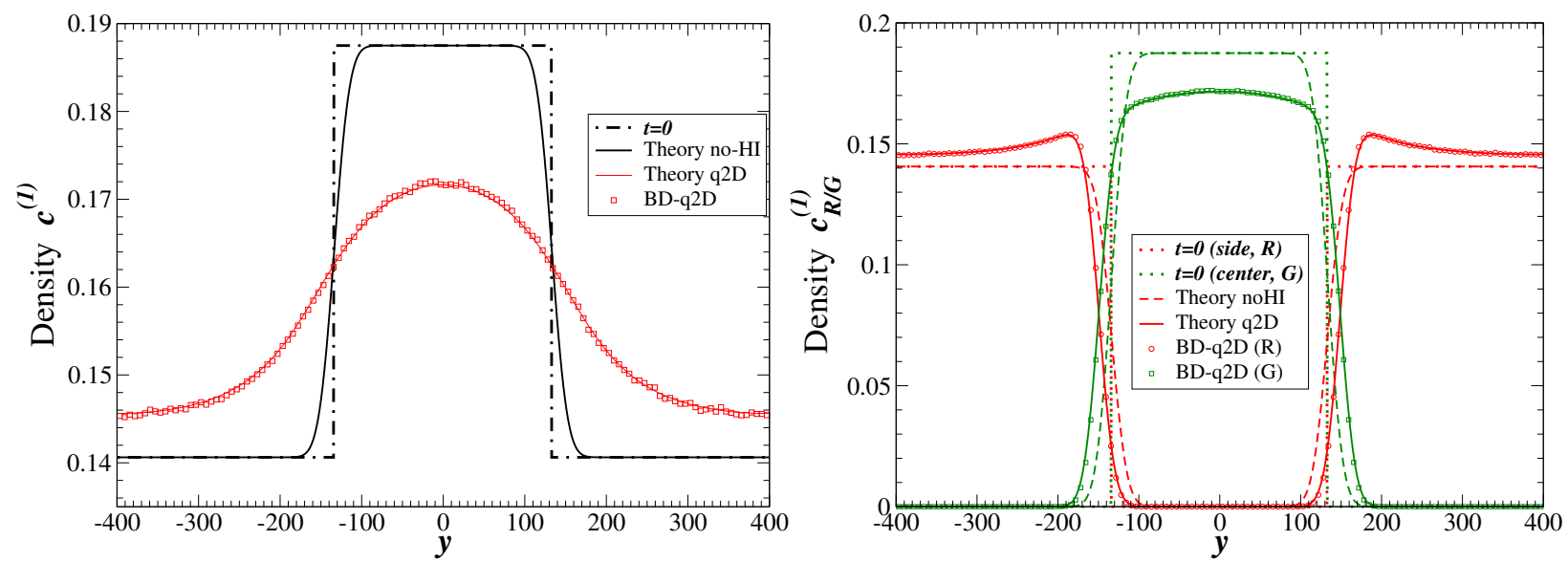

Figure 2: Ensemble-averaged one-dimensional density profiles illustrating the diffusion of a density jump in the $y$ coordinate. The density is initially higher in a stripe along the $x$ axes covering the middle third of the domain. Parameters are based on Setup A (see Table I) . (Left) The total density $c^{(1)}(y, t)$ at time $t=0$ (dashed-solid black line) and at time $t=2100$ for BD-q2D (red squares). Numerical solutions of the mean-field equation (30) are shown with a solid red line. The solution of the standard diffusion equation, which applies without hydrodynamic interactions, is shown with a solid black line. (Right) Density of "red" particles $c_{R}^{(1)}(y, t)$ (red lines and red circles) and "green" particles $c_{G}^{(1)}(y, t)$ (green lines and green squares) at time $t=0$ (dotted lines) and at $t=2100$ for BD-q2D (symbols). The numerical solution of (31) is shown with a solid line of the same color as the corresponding symbols. For comparison we also show the exact solution without hydrodynamics with a dashed line of the same color.

takes the form of two coupled nonlinear nonlocal diffusion equations,

$$
\partial_{t} c_{R / G}^{(1)}(\boldsymbol{r}, t)=\boldsymbol{\nabla} \cdot\left(\chi \nabla c_{R / G}^{(1)}(\boldsymbol{r}, t)+\left(k_{B} T\right) c_{R / G}^{(1)}(\boldsymbol{r}, t) \int \mathcal{R}\left(\boldsymbol{r}, \boldsymbol{r}^{\prime}\right) \boldsymbol{\nabla}^{\prime} c^{(1)}\left(\boldsymbol{r}^{\prime}, t\right) d \boldsymbol{r}^{\prime}\right)
$$

where the subscript $\mathrm{R} / \mathrm{G}$ labels the species by color and $c^{(1)}=c_{R}^{(1)}+c_{G}^{(1)}$. If we add these two equations we get back equation (30), as we must.

For the setup studied in the left panel of Fig. 2, we color all of the particles in the central stripe $-L_{y} / 6 \leq y \leq L_{y} / 6$ green, and color the remaining particles red. We compare ensemble-averaged results from BD-q2D simulations with a numerical solution of (31) in the right panel of Fig. 2. Without hydrodynamics we get familiar diffusive mixing with Gaussian tails, as illustrated with dashed lines in the figure. For Quasi2D hydrodynamics, however, we see that the the red particles are pushed away by the excess of green particles 

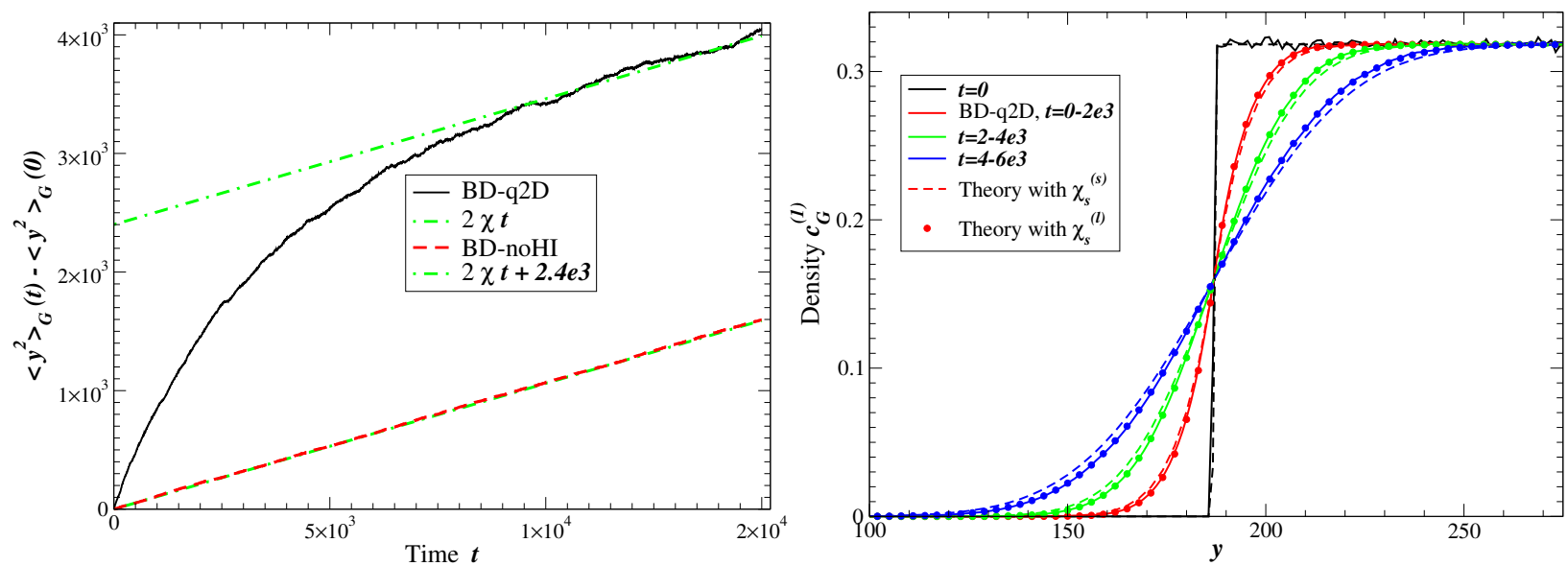

Figure 3: (Left) Mean squared displacement $\left\langle y^{2}\right\rangle_{G}(t)-\left\langle y^{2}\right\rangle_{G}(0)$ (see (32)) along the $y$ axis for the "green" particles, i.e., the ones initially at a higher density in the middle third stripe of the domain, for the same simulations used to produce Fig. 2. Without hydrodynamics the MSD follows the familiar diffusive evolution $2 \chi t$ at all times. For Quasi2D the MSD initially grows much more rapidly due to the collective interactions, but eventually settles to the expected diffusive growth at long times. (Right) Ensemble-averaged density of "green" particles $c_{G}^{(1)}(y)$ illustrating the diffusive mixing of red and green particles in Quasi2D, starting from a uniform density with a perfectly sharp red-green interface at $t=0$ (black lines). Parameters are based on Setup B (see Table I). The density is averaged over time intervals $0<t \leq 2 \cdot 10^{3}$ (red lines), $2 \cdot 10^{3}<t \leq 4 \cdot 10^{3}$ (green lines) and $4 \cdot 10^{3}<t \leq 6 \cdot 10^{3}$ (blue lines). Theoretical results based on the analytical solution of the standard diffusion equation are shown with dashed lines for $\chi=\chi_{0}$, and with solid circles for $\chi=\chi_{s}^{(l)} \approx 0.85 \chi_{0}$.

in the middle stripe. We find that it is $c_{R}^{(1)}$ that shows the fat power-law $y^{-3}$ tails, while $c_{G}^{(1)}$ has rapidly decaying Gaussian tails (not shown). This can also be appreciated by computing the second moment

$$
\left\langle y^{2}\right\rangle_{G}(t)=\frac{\left\langle\int y^{2} c_{G}(y, t) d y\right\rangle}{\left\langle\int c_{G}(y, t) d y\right\rangle},
$$

which is finite and grows linearly in time for long times both with and without hydrodynamics, as shown in the left panel of Fig. 3 .

Taken together, these results demonstrate that particles still exhibit only Gaussian diffusive displacements even in Quasi2D. Along the way, however, they effectively repel nearby particles, thus creating density waves that exhibit the unusual power-law behavior. Indeed, 
it has already been observed in [8, 9] that if one places a localized density perturbation in an otherwise empty domain, the tails are Gaussian rather than power law, since there are no particles to "push" away and thus create the density wave. In particular, if we color particles in an otherwise uniform suspension, $c^{(1)}(y)=c_{0}=$ const., the nonlinear convolution term in (31) disappears and one obtains a pair of uncoupled diffusion equations, despite the fact that red and green particles still "repel" each other with the long-ranged $1 / r$ hydrodynamic interaction 10 .

We explore this claim further in Fig. 3 by performing numerical experiments using parameters from Setup B (see Table【), $\phi=1$, except that we have used a small time step size $\Delta t=0.01$ to minimize temporal integration errors. See Fig. 9 for a visual representation of a single instance of the diffusive mixing. In the right panel of Fig. 3 we show simulation results for $c_{G}^{(1)}(y)$, averaged over 100 simulations and additionally averaged over time intervals of duration $2 \cdot 10^{3}$ in order to improve the statistics. We compare BD-q2D (solid lines) with the solution of an uncoupled system of two diffusion equations (dashed lines), one for each species. For the theory, we have used for the diffusion coefficient $\chi$ the short-time diffusion coefficient $\chi_{s}^{(s)}=\chi_{0}$ given by 22 . The agreement between simulation and theory is significantly improved if we use instead the long-time self diffusion coefficient (circles), $\chi_{s}^{(l)} \approx 0.85 \chi_{0}$ at this density $\phi=1$, see Fig. 1 and Section V. This clearly demonstrates that the mean-field equations (30) and (31) are not exact even for a Quasi2D ideal gas of particles because collective density fluctuations play a non-negligible role in the dynamics of the ensemble average as well. Note that incorporating the time-dependent self diffusion coefficient observed in Fig. 1 in the DDFT equations would require introducing memory (i.e., non-Markovian behavior), which is beyond the scope of this work.

\section{LONG-TIME SELF DIFFUSION}

In Fig. 1 we observed a notable decrease of the time-dependent diffusion coefficient from the short-time or bare value $\chi_{s}^{(l)}=\chi_{0}$ to a long-time or renormalized value $\chi_{s}^{(l)}$. This is due to a collective effect since the difference between the two values grows with the density, as we show in the left panel of Fig. 4 .

It is known that the time evolution of the MSD depends on the gradients of the mobility matrix $\boldsymbol{M}$. In particular, the theoretical series expansion for the MSD of a given particle $i$ 
(see $(3.24)$ in [46]) is

$$
\operatorname{MSD}_{i}(t) \approx 4 \chi_{0} t-t^{2}\left(k_{B} T\right)^{2}\left\langle\frac{\partial M_{i l}^{\alpha \beta}}{\partial r_{k}^{\gamma}} \frac{\partial M_{i k}^{\alpha \gamma}}{\partial r_{l}^{\beta}}\right\rangle+O\left(t^{3}\right)
$$

where we imply summation over all repeated indices except for $i$, and use Latin indices to denote particles and Greek ones to denote dimensions. The expression inside the average can be written as the trace of a positive definite matrix and therefore is always positive, i.e., for short times the effective diffusion coefficient is reduced from its bare value. By examining the form of the second term in (33) we can see that in Quasi2D within the Oseen approximation (15),

$$
\left(k_{B} T\right)^{2}\left\langle\frac{\partial M_{i l}^{\alpha \beta}}{\partial r_{k}^{\gamma}} \frac{\partial M_{i k}^{\alpha \gamma}}{\partial r_{l}^{\beta}}\right\rangle=4 \alpha\left(\frac{k_{B} T}{6 \pi \eta}\right)^{2}\left\langle\sum_{j} \frac{1}{r_{i, j}^{4}}\right\rangle \approx 4 \alpha\left(\frac{k_{B} T}{6 \pi \eta}\right)^{2} \int_{r=a}^{\infty} \frac{2 \pi c_{0} r}{r^{4}} d r=4 \alpha c_{0} \chi_{0}^{2} .
$$

The positive non-dimensional coefficient of proportionality $\alpha$ is non-trivial to compute explicitly, but is, in any case, a constant independent of density. In passing from a discrete sum to the integral above we could have used the FCM kernel to avoid the cutoff at $r=a$; however, the integral converges and the cutoff does not affect the result beyond the value of the unknown coefficient $\alpha$. The above relation predicts that for short times

$$
\operatorname{MSD}(t) \approx 4 \chi_{0} t-4 \alpha c_{0} \chi_{0}^{2} t^{2}=4 \chi_{0} t\left(1-\frac{\alpha \phi \chi_{0}}{\pi a^{2}} t\right)
$$

Linear fits to the MSD at short times give coefficients that are in good agreement with (34) providing $\alpha \approx 0.088$, see black squares in the right panel of Fig. 4 .

At longer times, however, the MSD strongly deviates from the quadratic approximation (34). We find that

$$
\frac{\operatorname{MSD}(t)}{4 \chi_{0} t}=1-\frac{\Delta}{\left(1+(\tau / t)^{b}\right)}
$$

accurately fits the numerical results, as shown in the left panel of Fig. 4. The fitting parameters shown in Fig. 5 include the exponent $b(\phi)$, the duration of the subdiffusive regime (cross-over time) $\tau(\phi)$, and the relative jump of the self diffusion coefficient $\Delta(\phi)=$ $1-\chi_{s}^{(l)} / \chi_{0}$. Interestingly, we find that $\Delta \approx\left(\tau_{s} / \tau(\phi)\right)^{1 / 2}$, as illustrated in the left panel of Fig. 5. Here the onset of the sub-diffusive regime $\tau_{s}=2.25$ is a constant independent of $\phi$, and is indicated with a large open square in the left panel of Fig. 4. On the other hand, the left panel of Fig. 5 shows that the overall decrease of the self-diffusion coefficient is in 

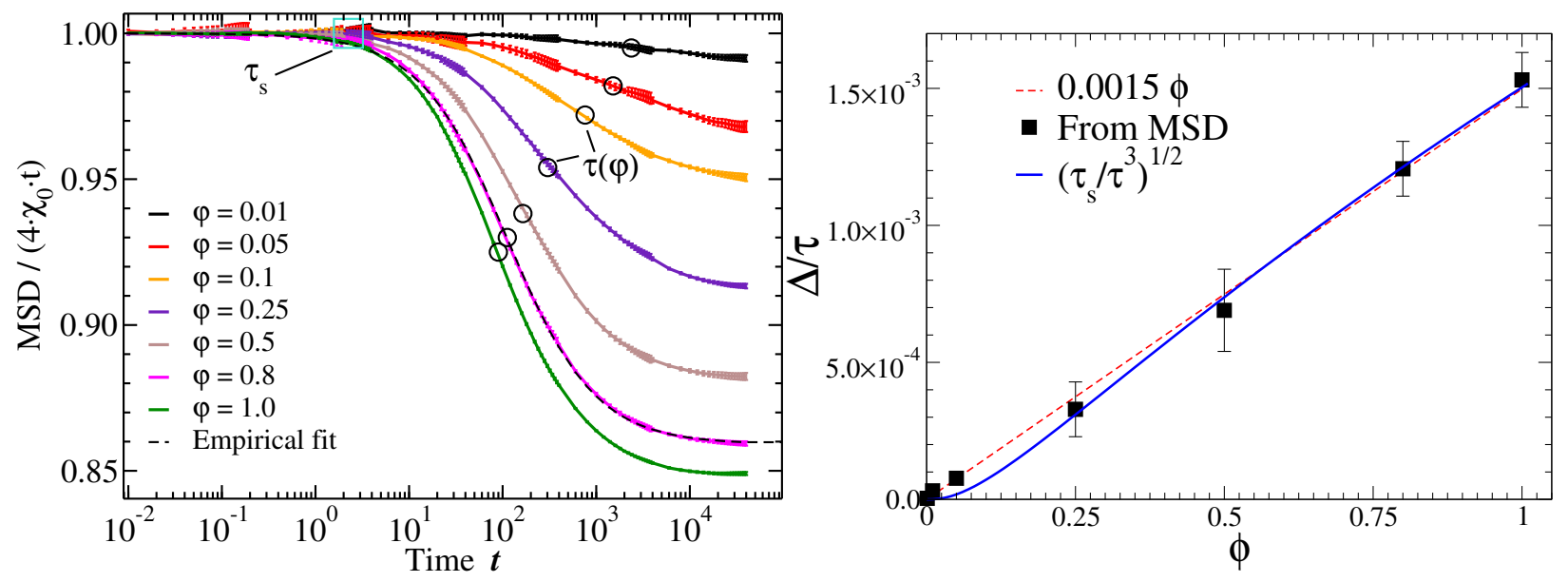

Figure 4: (Left) Mean square displacement of a tagged particle in Quasi2D for different packing densities $\phi$ (see legend). Each data set can be fit rather well by the empirical fit (35), as illustrated for $\phi=0.8$ (dashed line). Black circles denote the cross-over time $\tau(\phi)$, and the beginning of the subdiffusive regime $\tau_{s}$ is indicated with a cyan square. We have confirmed that finite size affects are negligible (not shown). (Right) The coefficient $\Delta / \tau$ appearing in the short-time expansion of the MSD (37) obtained in two different ways. The squares are obtained from linear fits to the short-time decay of the MSD shown in the left panel. The dashed red line confirms the linear relation with $\phi$ predicted by (34) with a fit coefficient giving $\alpha=0.088$. The solid blue line is obtained from the empirical fit $(36)$ and the empirical relation $\Delta=\left(\tau_{s} / \tau(\phi)\right)^{1 / 2}$. Both ways to obtain $\Delta / \tau$ prove to be consistent when the fit exponent $b \approx 1$ (see inset in left panel of Fig. 5).

reasonable agreement with the empirical fit

$$
\Delta \approx \phi_{c} \ln \left(1+\phi / \phi_{c}\right)
$$

where the fitting parameter $\phi_{c} \approx 0.0493$ is the surface fraction at which the collective renormalization of the diffusion coefficient becomes noticeable. Note that for $\phi<\phi_{c}$ the collective effects are quite small and our fits are subject to large statistical errors.

In order to connect the fit in (35) with the expansion in (34), we need to Taylor expand (35). The inset in the left panel of Fig. 5 shows that the exponent $b$ increases slowly from $b \approx 0.8$ for $\phi=0.05$ to $b \approx 1$ for $\phi=1$. If $b=1$, then the formula (35) is analytic and

$$
\operatorname{MSD}(t \ll \tau) \approx 4 \chi_{0} t\left(1-\frac{\Delta}{\tau} t\right)
$$



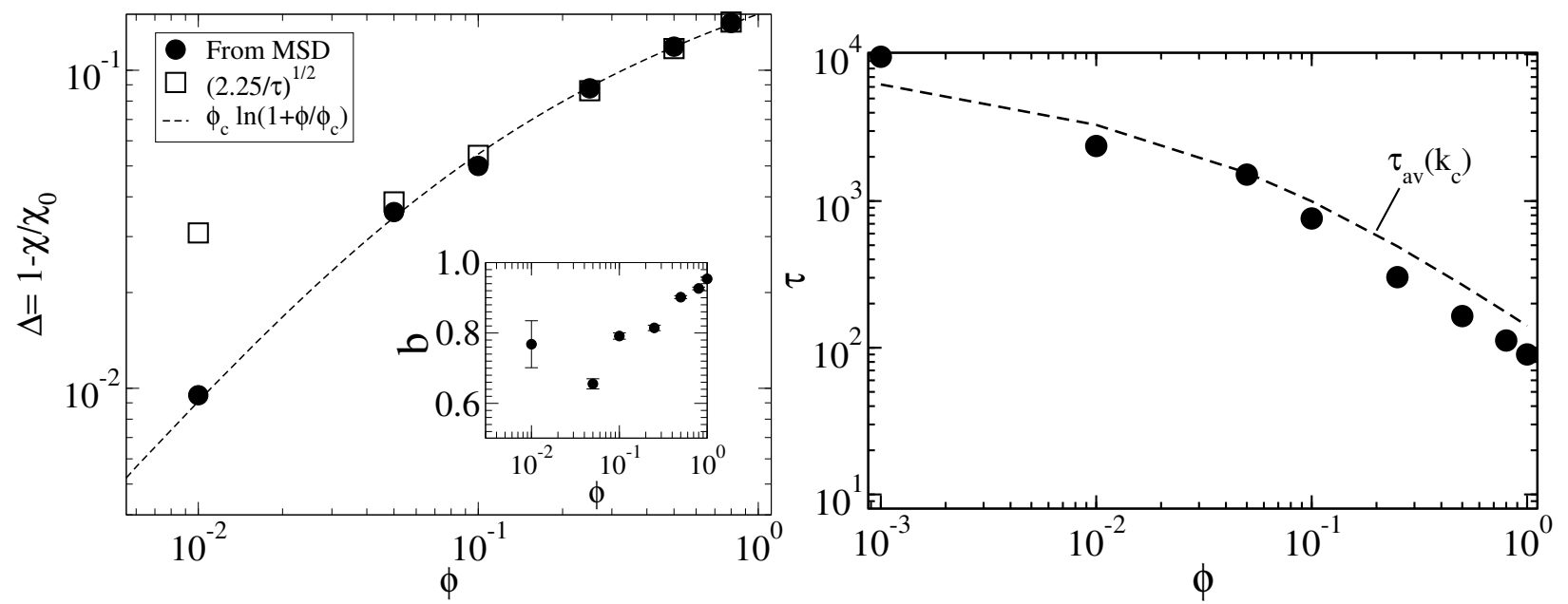

Figure 5: Parameters in the fit (35) to the MSD as a function of the surface packing fraction $\phi$. (Left) The relative decrease in the self-diffusion coefficient $\Delta$ (filled circles). The empty squares show the empirical relation $\Delta \approx\left(\tau_{s} / \tau(\phi)\right)^{1 / 2}$, where $\tau_{s}=2.25$. The dashed line is an empirical fit $\Delta=\phi_{c} \ln \left(1+\phi / \phi_{c}\right)$ with $\phi_{c}=0.0493$. The inset shows the exponent $b(\phi)$. (Right) The cross-over time $\tau$ for the subdiffusive regime (circles), compared to the average collective relaxation time $\tau_{c}\left(k_{c} ; \phi\right)$ defined in 39 (dashed line), where $k_{c}=\left(\alpha / \pi a^{2}\right)^{1 / 2}=0.17$.

Comparing (34) and (37), we conclude that

$$
\frac{\Delta}{\tau} \approx \frac{\alpha \chi_{0}}{\pi a^{2}} \phi=\chi_{0} k_{c}^{2} \phi
$$

where $\alpha$ is defined by (34). The right panel of Fig. 4 shows $\Delta / \tau$ computed by combining (36) with the relationship $\Delta=\left(\tau_{s} / \tau\right)^{1 / 2}$. The plot confirms an approximately linear relation between the relative drop in self-diffusion $\Delta$ and the duration of the diffusive regime $\tau$, consistent with the value of $\alpha$ estimated from the short-time behavior of the MSD. The relation 38 defines a "collective" length scale with wavenumber $k_{c} \equiv\left(\alpha / \pi a^{2}\right)^{1 / 2} \approx 0.17 a^{-1}$.

It is quite surprising to find a sub-diffusive regime in the dynamics of an ideal gas of non-interacting particles. This unexpected behavior arises because of collective density fluctuations, which lead to collective drift forces that affect the motion of a tagged particle. This suggests that $\tau(\phi)$ should be related to the relaxation time for collective density fluctuations at length scales smaller than or comparable to $k_{c}^{-1}$. We recall that in Quasi2D, the relaxation time of a density fluctuation of wavenumber $k$ is $\tau_{c}(k)=\left(\chi_{c} k^{2}\right)^{-1}$, where $\chi_{c}=\chi_{0}\left(1+\left(k L_{h}\right)^{-1}\right)$ with $L_{h}=2 a / 3 \phi$, see 15 . This leads us to an estimate of an 
average collective relaxation time,

$$
\tau_{\mathrm{av}}\left(k_{c}\right)=\frac{\int_{0}^{k_{c}} 2 \pi k \tau_{c}(k) S(k) d k}{\int_{0}^{k_{c}} 2 \pi k S(k) d k}=\frac{2}{\chi_{0} k_{c}^{2}} \ln \left(1+k_{c} L_{h}\right) .
$$

The right panel of Fig. 5 shows that the cross-over time $\tau$ extracted from the fits of the MSD agrees reasonably well with $\tau_{\text {av }}\left(k_{c}=0.17 a^{-1}\right)$. This confirms that the sub-diffusion of individual particles is related to the collective configurational memory of the system. Empirically we have found that the self diffusion of a particle is determined by collective density fluctuations of wavelengths smaller than about $2 \pi / k_{c} \approx 35 a$. A theoretical explanation of the empirical relations we have found is at present lacking, and requires generalizing the field theory presented in 24 to account for hydrodynamics, as we discuss in more detail in the Conclusions.

\section{FLUCTUATIONS IN TWO-DIMENSIONAL SYSTEMS}

In this section we examine the magnitude and dynamics of density fluctuations for diffusion confined to a plane, both at thermodynamic equilibrium and out of equilibrium. In particular, we look at the ensemble-averaged spectrum of the fluctuations at a given time $t$,

$$
\left.S(\boldsymbol{k} ; t)=\langle(\widehat{\delta} c(\boldsymbol{k}, t))(\widehat{\delta} c(\boldsymbol{k}, t)))^{\star}\right\rangle
$$

which we call the static structure factor. To simplify the notation, we usually omit the (potential) time dependence and denote the static structure factor with $S(\boldsymbol{k})$. The dynamics at steady state (either equilibrium or non-equilibrium) can be characterized by the dynamic structure factor

$S(\boldsymbol{k}, \omega)=\left\langle(\widehat{\delta c}(\boldsymbol{k}, \omega))(\widehat{\delta} c(\boldsymbol{k}, \omega))^{\star}\right\rangle, \quad$ or, equivalently, $\quad S(\boldsymbol{k}, t)=\left\langle(\widehat{\delta c}(\boldsymbol{k}, t))(\widehat{\delta c}(\boldsymbol{k}, 0))^{\star}\right\rangle$,

where $\omega$ is the wavefrequency. Here the average is both over time, and, since our system is ergodic, the steady-state ensemble. Note that $S(\boldsymbol{k}) \equiv S(\boldsymbol{k}, t=0)$.

In order to model density fluctuations, we add stochastic fluxes to (11) in the spirit of linearized fluctuating hydrodynamics (FHD) [18]. We begin with equilibrium fluctuations and then consider non-equilibrium fluctuations. 


\section{A. Equilibrium Fluctuations}

In this section we consider a uniform system with background number density $c_{0}$ at thermodynamic equilibrium. We first examine the density fluctuations and then extend the results to account for particle color (species labels).

\section{Structure Factor for Density}

Obtaining the linearized FHD equations from (11) is not trivial (see discussion in Sections 3.2 and 4.1 of [16]), however, the appropriate equations can essentially be guessed or at least inferred from fluctuation-dissipation balance. Basically, we know that at thermodynamic equilibrium (i.e., no macroscopic gradients), in the absence of direct particle interactions, we have no spatial correlations, $g_{2}(r)=1$ for all $r$, and $S(\boldsymbol{k})=c_{0}$ for all wavenumbers. From this we can infer that the linearized FHD equation for density at thermodynamic equilibrium is

$$
\begin{aligned}
\partial_{t} \delta c(\boldsymbol{r}, t) & =\chi \boldsymbol{\nabla}^{2} \delta c(\boldsymbol{r}, t)+\sqrt{2 c_{0} \chi} \boldsymbol{\nabla} \cdot \mathcal{W} \\
& +c_{0}\left(k_{B} T\right) \boldsymbol{\nabla} \cdot\left(\int \boldsymbol{\mathcal { R }}\left(\boldsymbol{r}-\boldsymbol{r}^{\prime}\right) \boldsymbol{\nabla}^{\prime} \delta c\left(\boldsymbol{r}^{\prime}, t\right) d \boldsymbol{r}^{\prime}\right)-c_{0}(\boldsymbol{\nabla} \cdot \boldsymbol{w}),
\end{aligned}
$$

where $\mathcal{W}(\boldsymbol{r}, t)$ is a standard white-noise Gaussian vector field with uncorrelated components. The first line in this equation is the standard linearized stochastic diffusion equation that would apply in the absence of hydrodynamics [15, 47], and it maintains fluctuationdissipation balance even if it weren't for the second line. The second term in the second line comes from the random advection term

$$
\boldsymbol{\nabla} \cdot(\boldsymbol{w} c)=c(\boldsymbol{\nabla} \cdot \boldsymbol{w})+\boldsymbol{w} \cdot \boldsymbol{\nabla} c
$$

in (7). Upon linearization at equilibrium, the second term $\boldsymbol{w} \cdot \boldsymbol{\nabla} c$ disappears; but it is this term that leads to the giant fluctuations out of equilibrium studied in Section VIB 1. It is important to recall that both (11) and (40) are approximate, not only because we have linearized the fluctuations but also because in Quasi2D the value of $\chi$ is ambiguous. In what follows we simply use the short-time self diffusion coefficient, but at larger length and time scales the somewhat smaller long-time self diffusion coefficient should be used. 
In Fourier space 40 reads

$$
\partial_{t}(\widehat{\delta c})=-\left(k^{2}+c_{0}\left(k_{B} T\right)(\boldsymbol{k} \cdot \widehat{\mathcal{R}} \cdot \boldsymbol{k})\right) \widehat{\delta c}+\sqrt{2 c_{0} \chi}\left(i k \mathcal{Z}_{\boldsymbol{k}}\right)-c_{0}(i \boldsymbol{k} \cdot \widehat{\boldsymbol{w}}),
$$

where $\mathcal{Z}_{\boldsymbol{k}}$ is a standard white-noise process (one per wavenumber) associated with $\widehat{\mathcal{W}}_{\boldsymbol{k}}$. Using the fact that $\left\langle\widehat{\boldsymbol{w}} \widehat{\boldsymbol{w}}^{\star}\right\rangle=2\left(k_{B} T\right) \widehat{\boldsymbol{\mathcal { R }}}$ and $\widehat{\boldsymbol{w}}$ and $\mathcal{Z}_{\boldsymbol{k}}$ are uncorrelated, we obtain the equilibrium spectrum

$$
S(\boldsymbol{k})=\left\langle|\widehat{\delta c}|^{2}\right\rangle=\frac{c_{0} \chi k^{2}+c_{0}^{2}\left(k_{B} T\right)(\boldsymbol{k} \cdot \widehat{\mathcal{R}} \cdot \boldsymbol{k})}{\chi k^{2}+c_{0}\left(k_{B} T\right)(\boldsymbol{k} \cdot \widehat{\mathcal{R}} \cdot \boldsymbol{k})}=c_{0},
$$

which is the correct result since the system is an ideal gas at thermodynamic equilibrium.

The dynamic structure factor is given from (42) by

$$
S(\boldsymbol{k}, t)=c_{0} \exp \left(-k^{2} \chi_{c}(k) t\right)
$$

where the collective diffusion coefficient $\chi_{c}$ is given in (13)24). In the left panel of Fig. 6 we show $S(\boldsymbol{k}, t)$ for several selected wavenumbers, rescaling the time by the theoretical prediction for $k^{2} \chi_{c}(k)$ to overlap the data. In the right panel of Fig. 6 we compare numerical measurements of the collective diffusion coefficient from the rate of decay of $S(\boldsymbol{k}, t)$ with the theoretical prediction; we see an excellent agreement over all wavenumbers. To within statistical accuracy, in the left panel of Fig. 6 we see a mono-exponential decay over the accessible time period for our ideal gas system, without a statistically-significant difference between short and long-time diffusion coefficients. Observe that because of the large relative value of the enhancement of the collective diffusion coefficient relative to the self-diffusion coefficient, it is difficult to see the difference between the short- and long-time self diffusion coefficients on this figure. To see this difference more clearly we consider dynamic structure factors for color fluctuations.

\section{Dynamic Structure Factor for Color}

The linearized FHD equations for the density of red/green particles can be inferred from (31) by accounting for stochastic mass fluxes in a manner that preserves fluctuationdissipation balance. At thermodynamic equilibrium,

$$
\begin{aligned}
\partial_{t} \delta c_{R / G}(\boldsymbol{r}, t) & =\chi \boldsymbol{\nabla}^{2} \delta c_{R / G}(\boldsymbol{r}, t)+\sqrt{2 c_{R / G} \chi} \boldsymbol{\nabla} \cdot \mathcal{W}^{(R / G)}-c_{R / G}(\boldsymbol{\nabla} \cdot \boldsymbol{w}) \\
& +\left(k_{B} T\right) \boldsymbol{\nabla} \cdot\left(c_{R / G} \int \boldsymbol{\mathcal { R }}\left(\boldsymbol{r}-\boldsymbol{r}^{\prime}\right) \boldsymbol{\nabla}^{\prime} \delta c\left(\boldsymbol{r}^{\prime}, t\right) d \boldsymbol{r}^{\prime}\right)
\end{aligned}
$$



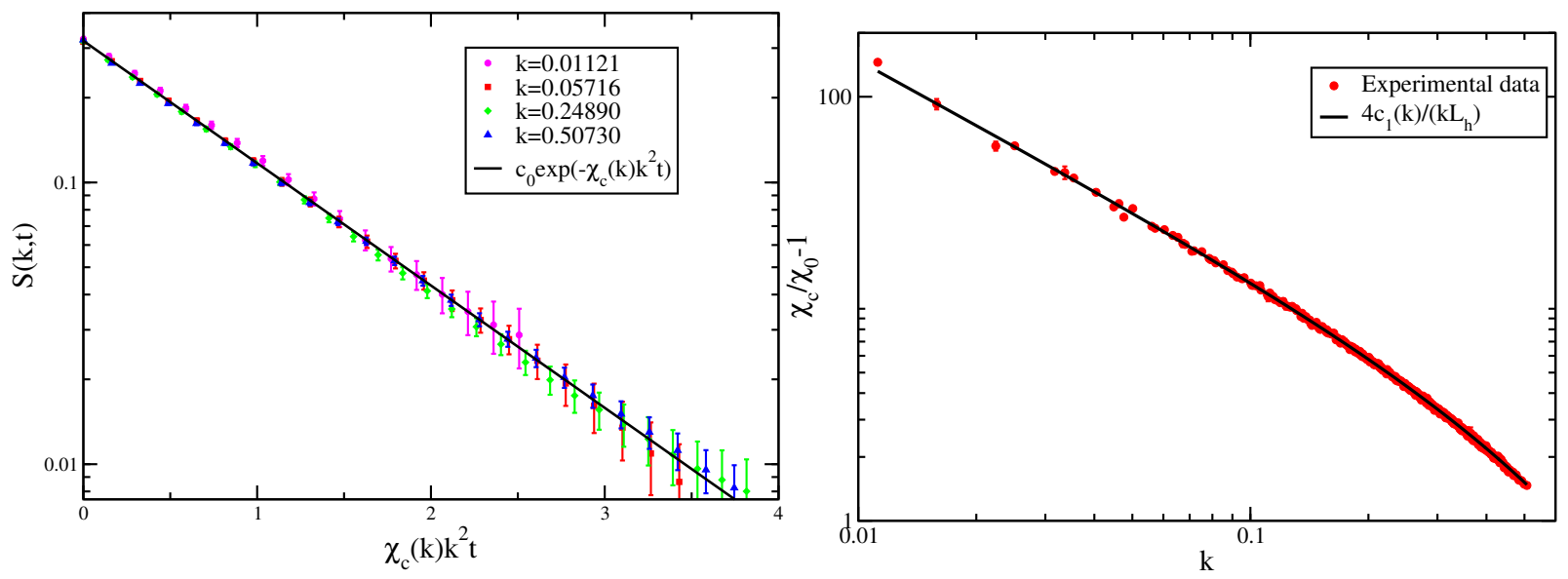

Figure 6: (Left) Numerical results from BD-q2D for dynamic structure factors for several wavenumbers (see legend), compared to the theoretical prediction (40) with $\chi_{c}(k)$ given in (24). The parameters used are taken from Setup B (see Table I), $\phi=1$, and the results are averaged over 1000 simulations, each of length $2 \cdot 10^{4}$ time steps. (Right) The collective diffusion coefficient estimated from the numerical dynamic structure factors shown in the left panel as a function of $k$ (symbols), compared to the theoretical prediction (24) (line). To estimate $\chi_{c}(k)$ we fitted $S(\boldsymbol{k}, t)$ to an exponential over the time interval during which the value decays by one order of magnitude.

where for notational simplicity we denoted the the average equilibrium color density with $c_{0}^{R / G} \equiv c_{R / G}$ without the subscript zero. The structure factor matrix, either static or dynamic, is defined as the covariance of the fluctuations of density in Fourier space,

$$
\boldsymbol{S}=\left[\begin{array}{l}
\left.\left.\left\langle\left(\widehat{\delta}_{R}\right)\left(\widehat{\delta}_{R}\right)\right)^{\star}\right\rangle\left\langle\left(\widehat{\delta}_{R}\right)\left(\widehat{\delta}_{G}\right)\right)^{\star}\right\rangle \\
\left.\left.\left\langle\left(\widehat{\delta c}_{G}\right)\left(\widehat{\delta}_{R}\right)\right)^{\star}\right\rangle\left\langle\left(\widehat{\delta c}_{G}\right)\left(\widehat{\delta c}_{G}\right)\right)^{\star}\right\rangle
\end{array}\right]=\left[\begin{array}{cc}
S_{R R} & S_{R G} \\
S_{G R} & S_{G G}
\end{array}\right] .
$$

From (44) it is straightforward to show that at thermodynamic equilibrium the static structure factor is

$$
\boldsymbol{S}(\boldsymbol{k})=\boldsymbol{S}_{0}=\left[\begin{array}{cc}
c_{R} & 0 \\
0 & c_{G}
\end{array}\right],
$$

which is consistent with an ideal gas mixture of red and green particles. To obtain the 
dynamic structure factors for color, we write (44) in Fourier space in matrix form,

$$
\begin{aligned}
\partial_{t}\left[\begin{array}{c}
\widehat{\delta c_{R}} \\
\widehat{\delta c_{G}}
\end{array}\right] & =-k^{2}\left[\begin{array}{cc}
\chi+\Delta \chi_{c}^{R} & \Delta \chi_{c}^{R} \\
\Delta \chi_{c}^{G} & \chi+\Delta \chi_{c}^{G}
\end{array}\right]\left[\begin{array}{l}
\widehat{\delta c_{R}} \\
\widehat{\delta c_{G}}
\end{array}\right]+\text { stochastic forcing }= \\
& =-k^{2} \boldsymbol{M}\left[\widehat{\widehat{\delta c_{R}}} \widehat{\widehat{\delta c_{G}}}\right] \text { + stochastic forcing, }
\end{aligned}
$$

where we introduced the collective diffusion enhancements for red and green particles via

$$
\Delta \chi_{c}^{R / G}(k)=\chi\left(\frac{4 c_{1}(k a)}{k L_{h}^{R / G}}\right) \approx \frac{k_{B} T}{4 \eta k} c_{R / G},
$$

where $L_{h}^{R / G}=2 /\left(3 \pi a c_{R / G}\right)$. It is a standard result from 45 that

$$
\boldsymbol{S}(\boldsymbol{k}, t)=\exp \left(-\boldsymbol{M} k^{2} t\right) \boldsymbol{S}_{0}
$$

Computing the matrix exponential for an equimolar mixture, $c_{R}=c_{G}=c_{0} / 2$, gives

$$
\begin{aligned}
& S_{R R}(k, t)=S_{G G}(k, t)=\frac{c_{0}}{4}\left(\exp \left(-\chi_{c} k^{2} t\right)+\exp \left(-\chi k^{2} t\right)\right) \\
& S_{R G}(k, t)=S_{G R}(k, t)=\frac{c_{0}}{4}\left(\exp \left(-\chi_{c} k^{2} t\right)-\exp \left(-\chi k^{2} t\right)\right) .
\end{aligned}
$$

Observe that the structure factors are sums of two modes, one slow one related to the self-diffusion coefficient $\chi$, and one faster one related to the enhanced collective diffusion coefficient $\chi_{c}$.

If we label with a "red" tag only a small fraction of the particles, $c_{R} \ll c_{G} \approx c_{0}$, and only trace the red particles, we get

$$
S_{R R}\left(\boldsymbol{k}, t ; c_{R} \ll c_{G}\right) \approx c_{R} \exp \left(-\chi k^{2} t\right)
$$

which allows us to focus on the self-diffusion contribution. In Fig. 7 we show numerical results from BD-q2D for $S_{R R}(\boldsymbol{k}, t)$ when only 1/16-th of the particles are colored red. The results in Fig. 7 show that there is a transition from exponential decay with rate set by the short-time self-diffusion coefficient $\chi_{0}$ at short times, to a decay with rate set by the long-time self diffusion coefficient $\chi_{s}^{(l)}$ at long times. This means that even at the level of linearized FHD there are collective memory effects that arise due to renormalization of the transport coefficient by fluctuations. In particular this means that the equations (40) and (44) are not exact even for an ideal gas mixture, even at the linearized level, i.e., at the level of a Gaussian approximation for the fluctuations. 


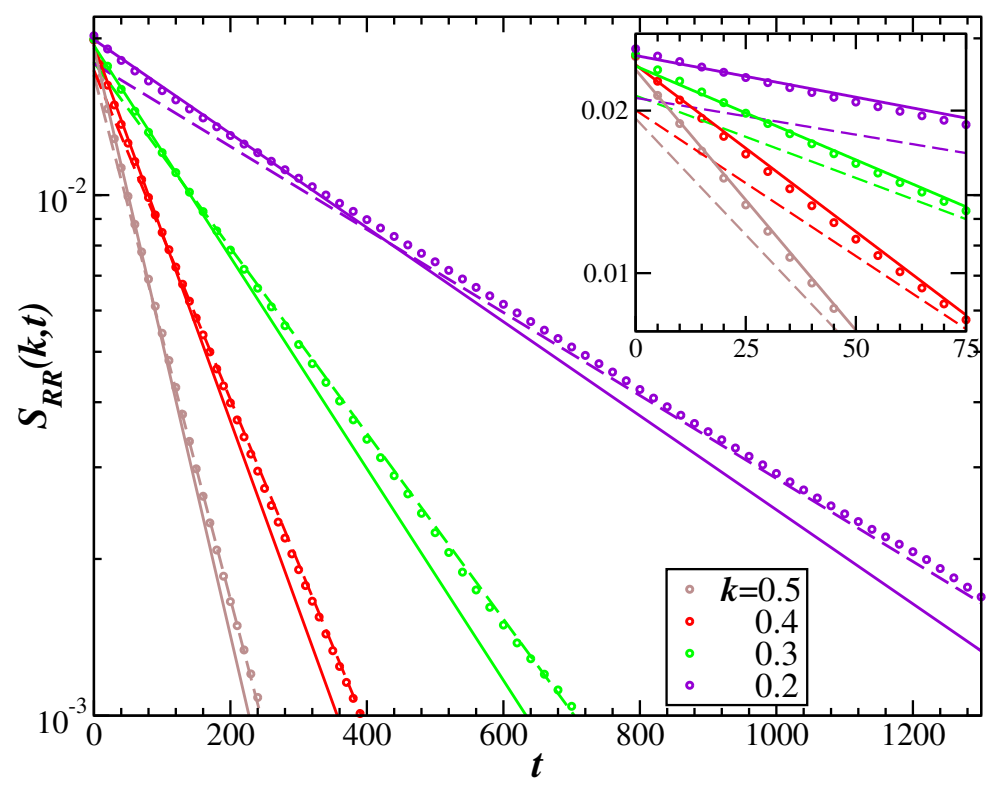

Figure 7: Dynamic structure factor for the density of red particles when a small fraction $c_{R} / c_{0}=$ 1/16 are colored red. The solid lines show the theoretical prediction (3) with $\chi$ taken to be the short-time self diffusion coefficient $\chi_{0}$, while the dashed lines use the long-time value $\chi_{s}^{(l)}$ (see Section $\mathrm{V}$ ). The inset focuses on short times. The parameters are the same as for Fig. 6 (Setup B, $\phi=1$, see Table I).

\section{B. Giant Non-equilibrium Fluctuations}

It is well known that nonequilibrium fluctuations in diffusive systems are long-ranged and strongly enhanced (giant) compared to the short-ranged equilibrium fluctuations [18]. These giant fluctuations have been measured experimentally in three dimensions in microgravity [19], but have not yet been measured in two-dimensional systems to our knowledge. Linearized fluctuating hydrodynamics predicts that in True2D the fluctuations are truly giant or "colossal" because the magnitude of the fluctuations is comparable to that of the mean, see Fig. 2 and associated discussion in [22]. The contribution from the compressibility of the fluid in the plane of confinement has not yet been evaluated because the theoretical calculations in [22] are based on the Saffman mobility kernel for membranes [2], which assumes that the flow is incompressible in the plane of the membrane.

In this section we study giant fluctuations in the presence of density and/or color gradients for a Quasi2D colloidal suspension confined to a planar interface, and compare to a True2D system such as a thin film in vacuum [21, 22]. We begin by considering a density gradient, 
and then consider a color gradient in the absence of a density gradient. In linearized FHD the general case of a density and color gradient is a simple superposition of the two cases we consider here.

In general, out of equilibrium the FHD equations should be linearized around the solution of the "deterministic" equations ${ }^{7}$. Here we take these unknown deterministic macroscopic equations to be the same as the approximate equations for the ensemble average (30)31). It is difficult to do better than this because to obtain the macroscopic equations we would have to perform a very nontrivial coarse graining in space and time [16]. The best approximation of the macroscopic equations known to us at present is (30)31] with $\chi$ being the long-time self-diffusion coefficient.

The linearization of the FHD equations around the (time-dependent) solution of the deterministic equations can often be performed numerically by artificially reducing the magnitude of the stochastic forcing terms [48]. Doing the same analytically is in general quite challenging [49]. Here we follow the traditional theoretical route [18] and assume that the macroscopic gradient is imposed externally and is constant in space and time. Furthermore, we assume that the gradient is sufficiently weak, so that the macroscopic density can be assumed not to vary in space. While in our numerical simulations the gradient varies both in space and time, this theoretical approximation enables simple estimates that give us the basic physical picture. For a more detailed comparison we numerically solve the FHD equations in Section VIB3.

\section{Density Gradient}

In this section we consider a weak imposed macroscopic gradient $\boldsymbol{\nabla} c_{0}$ of the total number density. In the presence of the weak gradient the linearized FHD equation (40) has an extra term $\boldsymbol{w} \cdot \boldsymbol{\nabla} c_{0}$ coming from (41),

$$
\begin{aligned}
\partial_{t} \delta c(\boldsymbol{r}, t) & =\chi \nabla^{2} \delta c(\boldsymbol{r}, t)+\sqrt{2 c_{0} \chi} \boldsymbol{\nabla} \cdot \mathcal{W} \\
& +c_{0}\left(k_{B} T\right) \boldsymbol{\nabla} \cdot\left(\int \boldsymbol{\mathcal { R }}\left(\boldsymbol{r}-\boldsymbol{r}^{\prime}\right) \boldsymbol{\nabla}^{\prime} \delta c\left(\boldsymbol{r}^{\prime}, t\right) d \boldsymbol{r}^{\prime}\right)-c_{0}(\boldsymbol{\nabla} \cdot \boldsymbol{w})-\boldsymbol{w} \cdot \boldsymbol{\nabla} c_{0}
\end{aligned}
$$

\footnotetext{
7 The precise statement is that the linearized FHD equations give the central limit theorem and describe the Gaussian fluctuations from the law of large numbers for the diffusive process.
} 
Let us consider a gradient in the $y$ direction, $\boldsymbol{g}=\boldsymbol{\nabla} c_{0}=g \hat{\boldsymbol{y}}$, and take a wavenumber perpendicular to the gradient, $\boldsymbol{k} \perp \boldsymbol{y}$. In this case, in Fourier space (47) becomes

$$
\begin{aligned}
\partial_{t}(\widehat{\delta c}) & =-\left(\chi k^{2}+c_{0}\left(\frac{k_{B} T}{\eta}\right) k c_{1}(k a)\right) \widehat{\delta c} \\
& +\sqrt{2 c_{0} \chi}\left(i k \mathcal{Z}_{\boldsymbol{k}}\right)-i c_{0} \sqrt{\frac{2\left(k_{B} T\right) k c_{1}(k a)}{\eta}} \mathcal{Z}_{\boldsymbol{k}}^{(1)} \\
& -g \sqrt{\frac{2\left(k_{B} T\right) c_{2}(k a)}{\eta k}} \mathcal{Z}_{\boldsymbol{k}}^{(2)}
\end{aligned}
$$

where $\mathcal{Z}_{\boldsymbol{k}}^{(1 / 2)}$ are independent standard white-noise processes (one per wavenumber) associated with the vortical/longitudinal modes of $\boldsymbol{w}$, see (27). From this equation we obtain the static structure factor

$$
S(k)=\left\langle|\widehat{(\delta c)}|^{2}\right\rangle=S_{0}+\Delta S=c_{0}+g^{2} \frac{\pi a^{2} c_{2}(k a)}{k^{2}\left(\pi a^{2} \beta k+c_{1}(k a) \phi\right)},
$$

where $\beta$ is defined in (23). The second term above represents the nonequilibrium fluctuations in excess of the equilibrium spectrum $S_{0}=c_{0}$.

To get an idea of how big the nonequilibrium contribution may be, let's consider small wavenumbers $k a \ll 1$, to get

$$
\frac{\Delta S}{S_{0}}(k a \ll 1) \approx g^{2} \begin{cases}\frac{2}{c_{0}^{2}} \cdot \frac{1}{k^{2}} & \text { for Quasi2D } \\ \frac{4 \pi}{c_{0} \ln \left(\frac{L}{3.71 a}\right)} \cdot \frac{1}{k^{4}} & \text { for True2D. }\end{cases}
$$

The $1 / k^{4}$ divergence for True2D is well-known and leads to "giant" nonequilibrium fluctuations [16, 22]. For Quasi2D the divergence is only $1 / k^{2}$. To get an idea of the magnitude of the nonequilibrium correction, let us assume that the gradient is imposed with boundary conditions over a length scale $L$ comparable to the size of the system, $g=c_{0} / L$, and consider a small wavenumber $k=2 \pi / L$. Denoting $L / a=N_{L}$, we estimate

$$
\max \frac{\Delta S}{S_{0}} \approx \begin{cases}\frac{1}{2 \pi^{2}} \ll 1 & \text { for Quasi2D } \\ \frac{N_{L}^{2} \phi}{4 \pi^{4} \ln \left(N_{L} / 3.71\right)} \gg 1 & \text { for True2D. }\end{cases}
$$

We conclude that in Quasi2D it is not possible to measure the nonequilibrium fluctuations since they are much smaller than equilibrium fluctuations for all wavenumbers. This is in agreement with numerical observations that there are no giant fluctuations in Quasi2D, as illustrated in the top row of Fig. 8. In this numerical experiment, we use parameters from 
Setup B (see Table I), but double the system size in both directions. We place $\sim 1.333 \cdot 10^{5}$ particles only in the middle third stripe of the domain, giving an initial packing density $\phi=1$ in the middle and zero elsewhere. For Quasi2D (BD-q2D) we follow the evolution to time $T_{q 2 D}=6775$, and for True2D (BD-t2D) we follow the evolution to $T_{t 2 D}=791$. This ensures that at the final time the diffusive mixing has progressed to the same relative time, $\chi_{q 2 D} T_{q 2 D}=\chi_{t 2 D} T_{t 2 D}$. In Fig. 8 we show several snapshots of the density (time increases from left to right) during the diffusive spreading of the initial density perturbation. Visually we do not see any large-scale structure of the density fluctuations for Quasi2D. We have examined the spectrum of the fluctuations averaged along the $y$ directions, and have been unable to measure $\Delta S$ compared to $S_{0}$ to within statistical accuracy. In True2D, the particles have spread much less because of the absence of the collective "repulsion", however, we can already visually appreciate the diffusive growth of giant fluctuations. At later times (not shown) we observe fully-developed "colossal" fluctuations in True2D, as illustrated in the bottom row in Fig. 9 for a slightly different setup. 

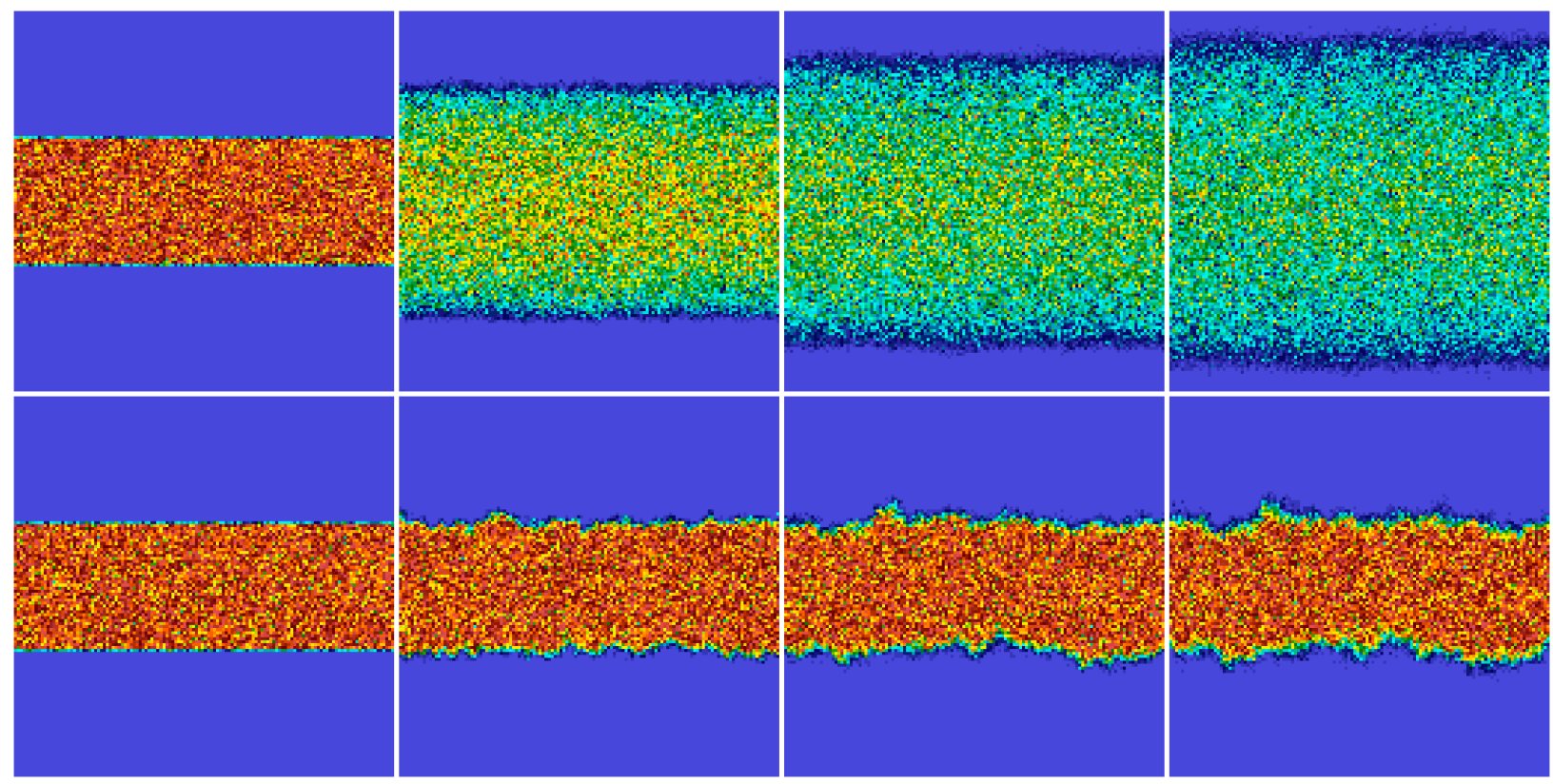

Figure 8: Diffusion of a density perturbation initially localized in the middle third of the domain.

We show snapshots at several points of equal relative time for BD-q2D (top row) and BD-t2D (bottom row). Snapshots are shown at times $0, T / 3,2 T / 3$ and $T$ (left to right), where the total simulation time $T$ is $T_{q 2 D}=6775$ and $T_{t 2 D}=791$. The images show the number density computed by counting the number of particles in each cell of a $128^{2}$ grid; the color bar is fixed to range from 0 (blue) to 0.4 (red) in all snapshots.

Note that in True3D the magnitude of the long-ranged nonequilibrium fluctuations is much larger than at equilibrium, in particular, in True3D one gets $\max \left(\Delta S / S_{0}\right) \sim \phi N_{L}^{2} \gg$ 1. Therefore, it is somewhat surprising that the giant fluctuations in the presence of a density gradient are strongly suppressed in Quasi2D, where the hydrodynamics is still three dimensional. It appears that the apparent long-ranged "repulsion" between the particles suppresses the fluctuations. This suggests that in order to see giant fluctuations we should eliminate density gradients so that the repulsion does not act, i.e., we should instead impose gradients of color (species label) only.

\section{Color Gradient}

In this section we consider fluctuations of color in the presence of a macroscopic color gradient without a gradient in density. We impose the gradient $\boldsymbol{\nabla} c_{R}=-\boldsymbol{\nabla} c_{G}=\boldsymbol{g}$ and 
perform the same computation as in the previous section. The linearized FHD equations consist of (44) with an additional forcing term $\pm \boldsymbol{w} \cdot \boldsymbol{g}$ on the right hand side. Considering wavenumbers perpendicular to the gradient, in Fourier space we have

$$
\begin{aligned}
\partial_{t}\left[\begin{array}{c}
\widehat{\delta c_{R}} \\
\widehat{\delta c_{G}}
\end{array}\right] & =-\left(\chi k^{2} \boldsymbol{I}+\left(\frac{k_{B} T}{\eta}\right) k c_{1}(a k)\left[\begin{array}{cc}
c_{R} & c_{R} \\
c_{G} & c_{G}
\end{array}\right]\right)\left[\begin{array}{c}
\widehat{\delta c_{R}} \\
\widehat{\delta c_{G}}
\end{array}\right]+i k\left[\begin{array}{c}
\sqrt{2 \chi c_{R}} \mathcal{Z}_{\boldsymbol{k}}^{(R)} \\
\sqrt{2 \chi c_{G}} \mathcal{Z}_{\boldsymbol{k}}^{(G)}
\end{array}\right] \\
& -i \sqrt{\frac{2\left(k_{B} T\right) k c_{1}(k a)}{\eta}}\left[\begin{array}{c}
c_{R} \\
c_{G}
\end{array}\right] \mathcal{Z}_{\boldsymbol{k}}^{(1)}-\sqrt{\frac{2\left(k_{B} T\right) c_{2}(k a)}{\eta k}}\left[\begin{array}{c}
g \\
-g
\end{array}\right] \mathcal{Z}_{\boldsymbol{k}}^{(2)},
\end{aligned}
$$

where $\mathcal{Z}_{\boldsymbol{k}}^{(R / G)}$ are independent scalar white noise processes associated with $\mathcal{W}^{(R / G)}$. Solving this linear system of Ornstein-Uhlenbeck equations we obtain

$$
\boldsymbol{S}(k)=\boldsymbol{S}_{0}+\Delta \boldsymbol{S}=\left[\begin{array}{cc}
c_{R} & 0 \\
0 & c_{G}
\end{array}\right]+g^{2} \frac{c_{2}(k a)}{\beta k^{3}}\left[\begin{array}{cc}
1 & -1 \\
-1 & 1
\end{array}\right],
$$

where $\beta$ is defined in (23). Note that, if we look at the total density $\delta c=\delta c_{R}+\delta c_{G}$, we only see equilibrium fluctuations, as expected,

$$
\left.\langle(\widehat{\delta c})(\widehat{\delta c}))^{\star}\right\rangle=S_{R R}+S_{G G}+S_{R G}+S_{G R}=c_{R}+c_{G}=c
$$

In True2D there is no difference between color gradient or density gradient since the particles are passive non-interacting tracers. Therefore, we focus now on Quasi2D. Our theory (50) predicts that the nonequilibrium fluctuations of color in Quasi2D have a spectrum $\sim 1 / k^{3}$, and might therefore be measurable in simulations or experiments, unlike the fluctuations of total density in the presence of a density gradient. Specifically, for small $k$ we predict a nontrivial correlation between the density fluctuations of red and green particles,

$$
\Delta S_{R G}(k a \ll 1) \approx-g^{2} \frac{3 \pi a}{k^{3}} .
$$

To estimate how large the color fluctuations may be, we again take $c_{R}=c_{G}=c_{0}, g=c_{0} / L$, $k=2 \pi / L$ and $L=N_{L} a$, to estimate

$$
\max \frac{-\Delta S_{R G}}{c_{0}} \approx \frac{3 \phi N_{L}}{8 \pi^{3}} .
$$

For $N_{L}=800$ and $\phi=0.5$, for example, we estimate $\max \left(\Delta S / S_{0}\right) \approx 5$, which is much larger than 1 but not nearly as large as the corresponding value $\max \left(\Delta S / S_{0}\right) \sim 150$ for True2D.

Unlike the case of a pure density gradient illustrated in Fig. 8, one can appreciate the giant color fluctuations in Quasi2D even visually. In Fig. 9 we show snapshots of the 
density of "green" particles (i.e., particles which are initially localized in the middle third of the domain) comparing uncorrelated walkers (BD-noHI, top row), Quasi2D (BD-q2D, middle row), and True2D (BD-t2D, bottom row). In these simulations, we use parameters from Setup B (see Table I), $\phi=1$, and start with a uniform system where we colored the particles in the middle third of the domain green. For Quasi2D (BD-q2D) we follow the evolution to time $T_{q 2 D}=1.505 \cdot 10^{5}$, and for True2D (BD-t2D) we follow the evolution to $T_{t 2 D}=2 \cdot 10^{4}$. This ensures that at the final time the diffusive mixing has progressed to the same relative time, $\chi_{q 2 D} T_{q 2 D}=\chi_{t 2 D} T_{t 2 D}$. For comparison we have also simulated uncorrelated walkers (BD-noHI) to the same relative time. A close examination of the figure reveals visibly enhanced large-scale fluctuations in the presence of Quasi2D hydrodynamics compared to no hydrodynamics, in agreement with the $1 / k^{3}$ theoretical prediction (50). For True2D we see the development of "colossal" fluctuations, the magnitude of which is comparable to the mean as predicted by linearized FHD [22]. Interestingly, however, the very fact that fluctuation are not small compared to the mean invalidates the linearized FHD theory used to predict the colossal fluctuations in the first place, as we demonstrate numerically in Section VIB2. 

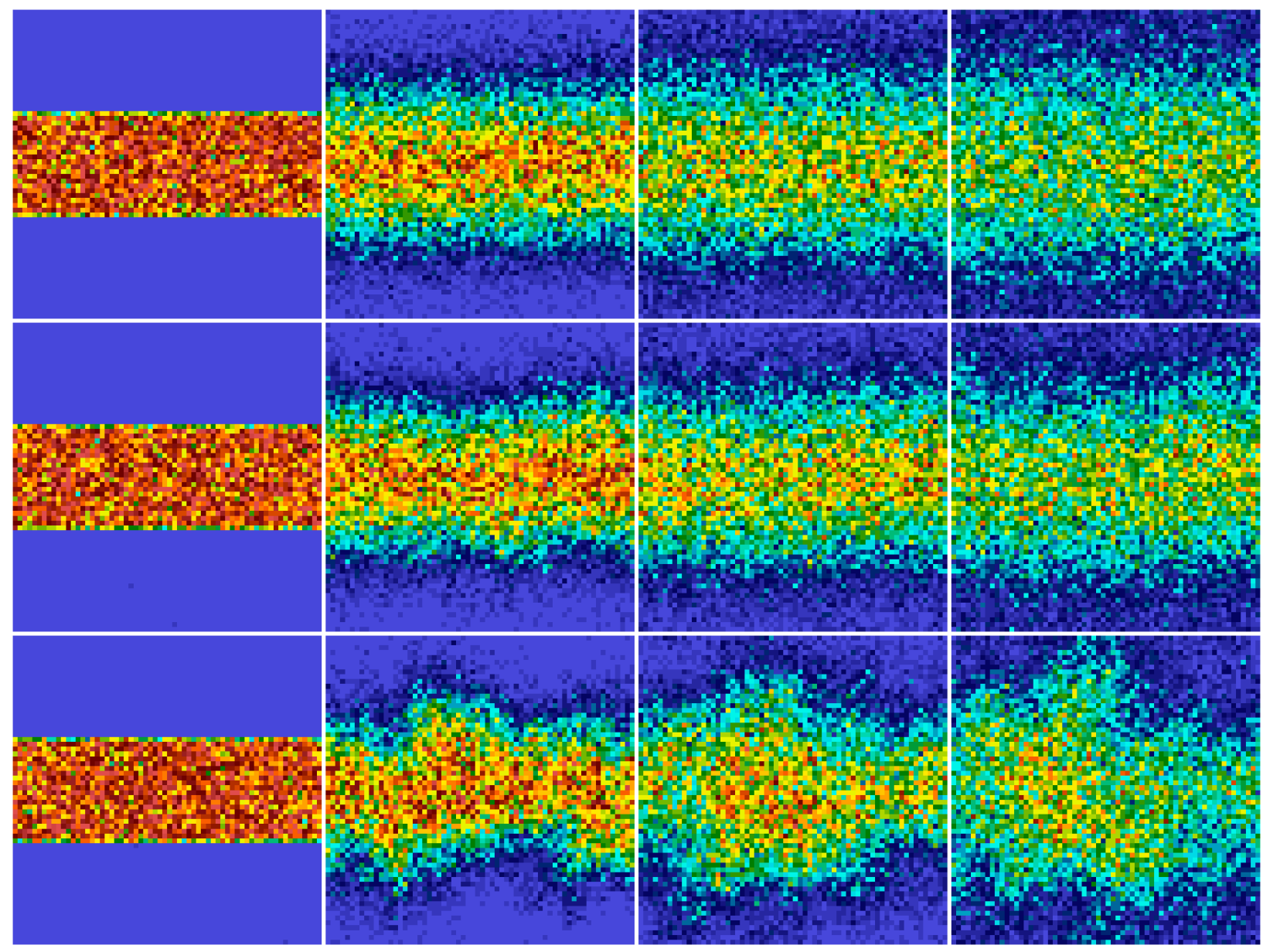

Figure 9: Diffusion of a perturbation of color (species) density in the absence of hydrodynamics (BD-noHI, top row), with Quasi2D hydrodynamics (BD-q2D, midde row), and with True2D hydrodynamics (BD-t2D, bottom row). The images show the number density of "green" particles computed by counting the number of green particles in each cell of a $64^{2}$ grid; the color bar is fixed to range from 0 (blue) to 0.4 (red) in all snapshots. The green particles are initially localized in the middle third of the domain and the rest of the domain is filled with red particles at the same packing density $\phi=1$, giving a uniform total density over the domain. We show several snapshots at times $0,0.3 T, 0.6 T$ and $0.9 T$ (left to right), where the total simulation time $T$ is $T_{q 2 D}=1.505 \cdot 10^{5}$ and $T_{t 2 D}=2 \cdot 10^{4}$.

For a more quantitative study of giant color fluctuations, in Fig. 10 we show the ensemble- and time-averaged structure factor $S_{R G}\left(k_{x}=k, k_{y}=0\right)$, i.e., the Fourier spectrum for wavenumbers perpendicular to the gradient, computed using the FFT on a grid of 512 cells. We average the spectrum separately over the first (time $0<t \leq T / 2$ ) and 

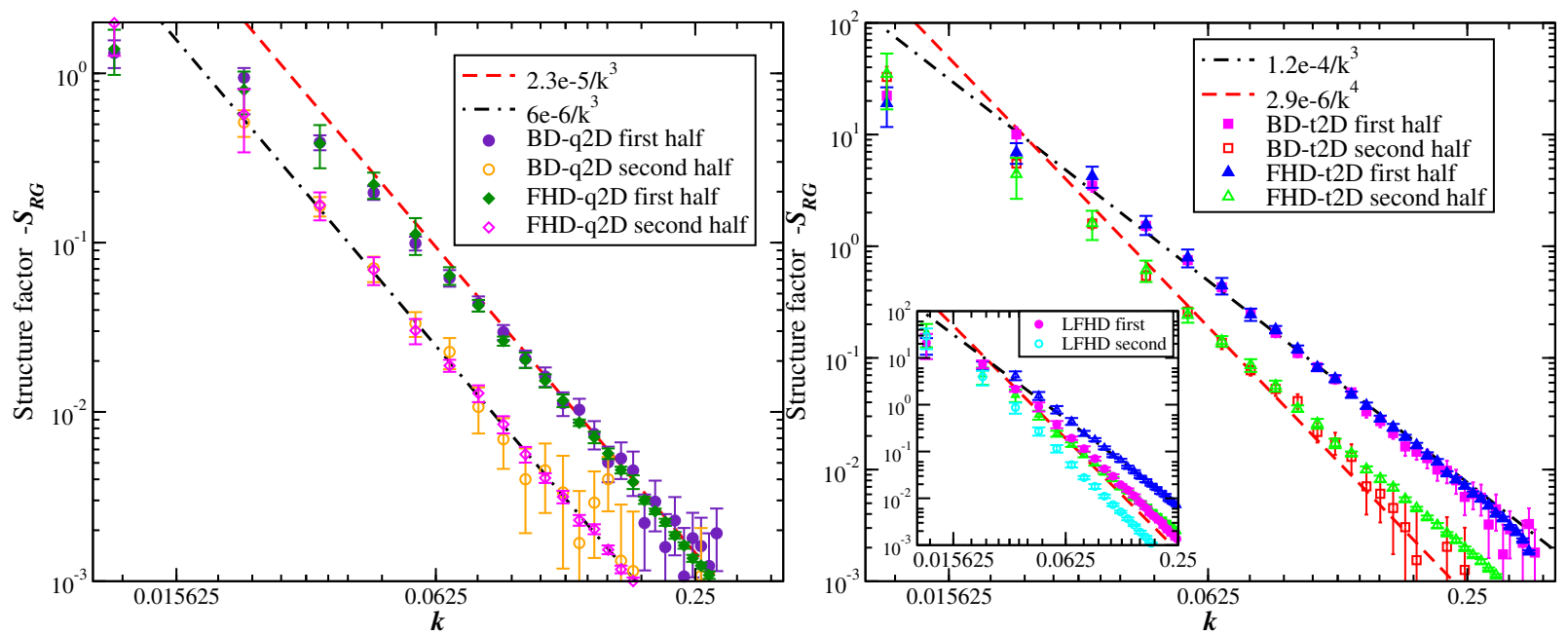

Figure 10: Spectrum of the fluctuations of color density during the diffusive mixing illustrated in Fig. 9, for Quasi2D (left) and True2D (right) hydrodynamics. The spectrum is averaged over the two halves of the simulations (filled symbols for first half and empty symbols for second half). We average the spectra over 64 simulations and show two standard deviation error bars. (Left) Results from BD-q2D (circles) for the first (solid circles) and second half (empty circles), compared to the $k^{-3}$ asymptotic power-law predicted by (4) (lines). Diamonds show results from a pseudo-spectral FHD solver for (53). (Right) Results from BD-t2D (squares) compared to results obtained by solving the nonlinear FHD equation (52) using a pseudo-spectral code (triangles) with an FFT grid of $64^{2}$ cells. The empirical power law $S_{R G} \sim-k^{-3}$ fits the data well over a broad range of wavenumbers. The inset demonstrates that solving the linearized FHD equations using the same pseudo-spectral code (circles) gives $S_{R G} \sim-k^{-4}$ in agreement with the theoretical prediction (50), but this prediction is in disagreement with the particle and nonlinear simulations.

second (time $T / 2<t \leq T$ ) halves of the simulation. In the first half of the simulation, the spectrum is still evolving toward its asymptotic power-law behavior but the fluctuations are larger because the gradient is largest initially. In the second half of the simulation, the spectrum has reached its asymptotic shape but the magnitude decays in time like the square of the gradient. Note that the fluctuations at the smallest wavenumbers grow slowest and are subject to strong finite size effects, and are therefore not expected to obey the prediction of the simple linearized FHD theory. 


\section{Nonlinear FHD equations}

In order to perform a more quantitative comparison between FHD predictions and results from the particle simulations shown in Fig. 10, we need to solve the FHD equations numerically for the time dependent diffusive mixing under consideration. This is straightforward to do in the linearized setting in True2D and True3D, and (numerically) linearized FHD simulations [48] favorably compare to experimental measurements for a three-dimensional system [20]. However, the right panel of Fig. 10 shows that results from BD-t2D (squares) deviate from the theoretical $k^{-4}$ decay over the first half of the simulation. This is because nonlinearities play a role since the fluctuations are comparable to the mean.

The nonlinear DDFT equations are formal and cannot be given a well-defined meaning as stochastic partial differential equations because their solution is a distribution and not a function. Instead, one must spatially coarse grain the dynamics in order to give nonlinear FHD meaning, as discussed at length in [15, 16, 50, 51]. However, we have been able to reproduce the particle results for the correlation between red and green particles (i.e., for $S_{R G}(k)$ ) by numerically solving (7) specialized for the case of an incompressible velocity field,

$$
\partial_{t} c_{R / G}=-\epsilon^{\frac{1}{2}} \boldsymbol{w} \cdot \nabla c_{R / G}+\chi \nabla^{2} c_{R / G}
$$

where we remind the reader that the advective term is to be interpreted in the Ito sense ${ }^{8}$. Here $\epsilon$ is a parameter that can be used to control the magnitude of the fluctuations [48] we define the structure factor of interest as $S_{R G}=\epsilon^{-1}\left\langle\left(\widehat{\delta c_{R}}\right)\left(\widehat{\delta c_{G}}\right)^{\star}\right\rangle$. Setting $\epsilon \ll 1$ makes the nonequilibrium fluctuations weaker and thus numerically linearizes the equations ${ }^{9}$. The initial condition we have used is $c_{R / G}(\boldsymbol{r}, t=0)=c_{R / G}^{(1)}(\boldsymbol{r}, t=0)$, which is a function and not a distribution like the initial condition (6) used in the particle simulations ${ }^{10}$. Because of this, 52 does not reproduce the equilibrium component of the fluctuations and instead

\footnotetext{
${ }^{8}$ For $\epsilon=1$, equation 52 can also be written as $\partial_{t} c_{R / G}=-\boldsymbol{w}_{h} \odot \nabla c_{R / G}$, where $\odot$ denotes a dot product interpreted in the Stratonovich sense [15, 16].

${ }^{9}$ in the limit $\epsilon \rightarrow 0$ one can give a precise meaning to $\sqrt[52]{52}$ in the spirit of linearized fluctuating hydrodynamics 48

${ }^{10}$ Our numerical experiments suggest that even for $\epsilon=O(1)$ the nonlinear equation $(52)$ is well-behaved and its solution remains a function and not a distribution, but it is difficult to make more precise statements at present.
} 
gives $S_{R R}=S_{G G}=-S_{R G}$, as predicted by the linearized theory (50) for the nonequilibrium contribution to the spectrum.

We solve the True2D nonlinear equations (52) numerically using a standard antialiased pseudo-spectral method; our MATLAB codes are available at https://github.com/ stochasticHydroTools/FHDq2D. In True2D the particles are passive tracers and there is no difference between short- and long-time self-diffusion coefficients, so we have set $\chi=\chi_{0}$. The results for $S_{R G}(k)$ for $\epsilon=1$ are shown with triangles in the right panel of Fig. 10. The FHD results are in excellent agreement with the results of the particle simulations (squares). The inset in the right panel of Fig. 10 shows that the numerical solutions of the linearized FHD equations, which we estimated using $\epsilon=0.01$, show a clear $-k^{-4}$ trend for both halves of the run (circles), and are not in agreement with the results from nonlinear FHD or BD-t2D. This suggests that in True2D one must numerically solve nonlinear FHD equations in order to quantitatively describe giant fluctuations.

In Quasi2D, it is significantly more challenging to write meaningful nonlinear FHD equations because of the nonlinearity of the convolution term arising due to the compressibility of the hydrodynamic kernel, as we discuss in more detail in the Conclusions. Instead, we have solved using a pseudospectral method a variant of (52) adapted to Quasi2D,

$$
\partial_{t} c_{R / G}=-\epsilon^{\frac{1}{2}} \boldsymbol{w}^{\perp} \cdot \nabla c_{R / G}+\chi \nabla^{2} c_{R / G},
$$

where $\boldsymbol{w}^{\perp}$ is the incompressible or vortical component of $\boldsymbol{w}$. More precisely, $\left\langle\boldsymbol{w}^{\perp}(\boldsymbol{r}, t) \otimes \boldsymbol{w}^{\perp}\left(\boldsymbol{r}^{\prime}, t^{\prime}\right)\right\rangle=\left(2 k_{B} T\right) \boldsymbol{\mathcal { R }}^{\perp}\left(\boldsymbol{r}-\boldsymbol{r}^{\prime}\right) \delta\left(t-t^{\prime}\right)$, where in Fourier space $\hat{\mathcal{R}}_{\boldsymbol{k}}^{\perp}=$ $\left(c_{2}(k a) / \eta k^{3}\right) \boldsymbol{k}_{\perp} \otimes \boldsymbol{k}_{\perp}$ is the incompressible component of the Quasi2D hydrodynamic kernel.

In the left panel of Fig. 10 we show numerical results for $S_{R G}(k)$ for $\epsilon=0.01$, but little change is observed if $\epsilon$ is further reduced or increased to the natural value $\epsilon=1$. This indicates that the equations are essentially linear and nonlinearities play little role. In our simulations we have used the (renormalized) long-time self-diffusion coefficient, $\chi=\chi_{l}^{(s)} \approx$ $0.86 \chi_{0}$, as suggested by the numerical results for the mean shown in the right panel of Fig. 3 , the matching is not as good if we use the short-time value $\chi=\chi_{0}$. We observe an excellent agreement between the results from BD-q2D and the numerical solution of (53) in the left panel of Fig. 10. This demonstrates that the nonequilibrium fluctuations in Quasi2D are controlled by the vortical component of the velocity, just as in True2D or True3D. This can be appreciated from the fact that the nonequilibrium contribution $\Delta S$ in (50) only involves 
$c_{2}(k)$ and does not depend on the amplitude of the compressible or longitudinal component $c_{1}(k)$, which only affects the equilibrium contribution $\boldsymbol{S}_{0}$.

\section{CONCLUSIONS AND DISCUSSION}

We developed an efficient algorithm for Brownian dynamics with hydrodynamic interactions, suitable for modeling diffusion of spherical colloids of hydrodynamic radius $a$ confined to a two-dimensional plane. We used this algorithm to perform large-scale particle simulations and studied collective diffusion on fluid-fluid interfaces (Quasi2D) and in twodimensional liquids (True2D). The nonzero compressibility of the three dimensional flow at the fluid-fluid interface leads to a nonzero divergence of the mobility matrix. Under the action of hydrodynamic fluctuations in the fluid, this compressibility acts like a pairwise repulsive potential of order $k_{B} T(a / r)$, and changes the nature of diffusion dramatically.

\section{A. Summary of Findings}

We first examined the evolution of ensemble averages of the particle number density. Consistent with prior studies, we obtained good agreement between a simple closure for the equations of dynamic density functional theory and particle simulations of an ideal gas of hydrodynamically-correlated Brownian particles. At the same time, we found that the effective particle-particle repulsion leads to a nontrivial reduction of the long-time selfdiffusion coefficient as the packing density increases, even for an ideal gas of non-interacting particles. By coloring the particles with two species labels, we elucidated the mechanism by which an initially localized density perturbation develops inverse-cubed power-law tails at later times, even though individual particles still have Gaussian displacements at long times consistent with ordinary diffusion. We found that the diffusive mixing of two colors is consistent with simple diffusion but with a diffusion coefficient equal to the long-time selfdiffusion coefficient, indicating that fluctuations renormalize the equations for the ensemble average and must be retained in a quantitatively-accurate description.

We further examined the magnitude and dynamics of collective density fluctuations at thermodynamic equilibrium and out of equilibrium. We found that for an ideal gas mixture, equilibrium dynamic structure factors show nearly mono-exponential decay, with the differ- 
ence between short and long times coming mainly from the difference between the short- and long-time self-diffusion coefficient. Out of equilibrium, we found that nonequilibrium density fluctuations are small compared to equilibrium fluctuations, indicating that in Quasi2D each instance of the diffusive spreading of density perturbations looks similar to the ensemble average. By contrast, in True2D nonequilibrium fluctuations grow to be comparable to the mean and make each instance of the mixing process qualitatively different from the ensemble average. We found that nonequilibrium color fluctuations exhibit a power-law spectrum typical of giant fluctuations seen in other geometries, but are much smaller in magnitude in Quasi2D than in True2D. Fluctuating hydrodynamics can accurately model the giant color fluctuations in both Quasi2D and True2D, with nonlinearities playing a significant role only in True2D. We found that the compressibility of the hydrodynamic tensor in Quasi2D does not affect giant color fluctuations in the absence of a density gradient; the magnitude of the red-green correlations could be accurately predicted by linearized FHD based on an incompressible fluid velocity. At the same time, we found that the hydrodynamic "repulsion" among the particles strongly suppresses density fluctuations in the presence of a density gradient. The difference between True2D and Quasi2D is striking and emphasizes the important role of hydrodynamics and fluctuations in diffusion in liquids. In True2D the mean is the same as in the absence of hydrodynamics, but the fluctuations dominate the mean and exhibit a yet unexplained $k^{-3}$ power law. In Quasi2D, the mean behavior differs strongly from standard diffusion, but the fluctuations are small compared to the mean and well-described by linearized fluctuating hydrodynamics.

In Section V we examined in some detail the time dependence of the self-diffusion coefficient, i.e., the mean square displacement. We found that the reduction of the self-diffusion coefficient with time is a collective effect governed by density fluctuations at length scales notably larger than the particle size. It remains a challenge to develop a theoretical understanding of this effect. In [24], following prior theoretical work of Demery and Dean [52, 53], the authors have developed a theory for the effective diffusion of a tracer particle in a dense suspension of soft repulsive colloids, in the absence of hydrodynamics. This theory predicts a reduction of the diffusion coefficient for repulsive soft spheres, just as we observe in Quasi2D, consistent with the interpretation of $(10)$ as a soft repulsive potential ${ }^{11}$. In

$\overline{11}$ The $1 / r$ behavior only applies in the far field and needs to be regularized at short distances. In our 
[24], the authors use fluctuating hydrodynamics to describe the collective fluctuations of the fluid excluding the tracer particle, and couple this bidirectionally to the equation of motion for the tracer particle itself. Generalizing this approach to Quasi2D diffusion is nontrivial. Importantly, our particles are correlated hydrodynamically and are driven not by independent noise but rather advected by a smooth in space and white in time random velocity field $\boldsymbol{w}$. Furthermore, our system is in detailed balance (time reversible) with respect to an ideal gas equilibrium distribution, unlike a gas of particles that interact with a direct repulsive potential.

\section{B. Future Directions}

It remains a challenge for the future to formulate nonlinear FHD equations in Quasi2D. A natural first guess is to consider a regularized (truncated) version of the formal fluctuating DDFT-HI equations [15],

$$
\begin{aligned}
\partial_{t} c_{R / G}(\boldsymbol{r}, t) & =\boldsymbol{\nabla} \cdot\left(-\boldsymbol{w}(\boldsymbol{r}, t) c_{R / G}(\boldsymbol{r}, t)+\chi \boldsymbol{\nabla} c_{R / G}(\boldsymbol{r}, t)+\sqrt{2 \chi c_{R / G}(\boldsymbol{r}, t)} \mathcal{W}^{(R / G)}(\boldsymbol{r}, t)\right) \\
& +\left(k_{B} T\right) \boldsymbol{\nabla} \cdot\left(c_{R / G}(\boldsymbol{r}, t) \int \boldsymbol{\mathcal { R }}\left(\boldsymbol{r}, \boldsymbol{r}^{\prime}\right) \boldsymbol{\nabla}^{\prime} c\left(\boldsymbol{r}^{\prime}, t\right) d \boldsymbol{r}^{\prime}\right)
\end{aligned}
$$

where we have now employed an Ito interpretation for the advective terms. Our attempts to solve (54) using a pseudospectral numerical method ${ }^{12}$ have failed to produce results in agreement with particle simulations (BD-q2D). The fluctuation-dissipation balance between the advection by the compressible (longitudinal) component of $\boldsymbol{w}$ (which acts as a source of fluctuations) and the nonlinear convolution term (which damps or dissipates fluctuations) is rather delicate and difficult to preserve in spatially coarse-grained equations.

In this work we focused on an ideal gas of non-interacting particles in order to emphasize the hydrodynamic effects. In any experimentally-realizable system, however, the particles will interact with each other with short-ranged steric forces at a minimum, and most likely with some additional longer-ranged interaction as well, such as partially-screened repulsive electrostatic forces [4] or attractive capillary forces [10]. Preliminary investigations for

formulation based on the FCM method, in Fourier space the apparent repulsive potential can be written as $\hat{U}(k)=(6 \pi a / k) c_{1}(k a)$.

${ }^{12}$ In our pseudo-spectral method, we simply truncate the Fourier modes at $k_{\max }=\pi / h$ where $h$ is the grid spacing, which is analogous to spatial discretization in finite volume methods. 
partially-confined Quasi2D suspensions [13] have already shown that the strong collective effects in Quasi2D diffusion persist in the presence of steric repulsion. At the same time, however, steric repulsion induces caging and introduces a new cage-breaking time scale. We therefore expect to see a strong difference between short and long times in both dynamic structure factors and nonequilibrium dynamics. Direct interactions can formally be included in the fluctuating DDFT-HI equations [15], however, the equations are no longer closed and making further progress requires introducing various approximations and density functionals inspired by static DFT [54, 55]. The errors made because of such approximations would in practice overwhelm the modest corrections to the self-diffusion coefficient we were able to isolate in this work by using an ideal gas.

Although we focused here on purely three-dimensional hydrodynamic interactions in an unbounded system, the same kind of compressibility effect likely plays a role in other geometries and systems as well. In particular, membranes immersed in fluid are another system where diffusion is confined to a surface but the hydrodynamics is three dimensional. Examples include thin smectic films in air [21] and lipid bilayers at the surface of vesicles. The Saffman model [56, 57] for membranes gives a modified form for the hydrodynamic correlation tensor (see Eq. (13) in the review [2]), which is consistent with our general form (25) with $f_{k}=0$ and $g_{k}=1 /\left(\eta k\left(k+k_{c}\right)\right)$. A number of modifications of these functions have been computed in slightly different geometries such as, for example, triply periodic systems [58]. Here $k_{c}$ is a roll-over wavenumber that depends on the ratio of the viscosity of the surrounding fluid to the two-dimensional viscosity of the membrane/film itself; for $k_{c}=0$ we get True2D hydrodynamics. The Saffman model assumes incompressibility in the plane, $f_{k}=0$, and therefore there would be no anomalous collective diffusion with Saffman hydrodynamic correlations; this justifies the simplified fluctuating hydrodynamic equations used in [22]. Much of the work on diffusion in membranes has focused on self-diffusion, and, to our knowledge, collective diffusion in membranes has not yet been carefully studied either experimentally or numerically. The True2D Brownian dynamics algorithm developed here can be used to study collective diffusion in membranes with a simple change of the function $g_{k}$, at least under the assumption that the Saffman model applies on the time and length scales of interest. Future work should probe the transition from Quasi2D hydrodynamics to Saffman-like hydrodynamics in dense monolayers of hard spheres on fluid-fluid interfaces, as well as in lipid bilayers. 
A nonzero divergence of the hydrodynamic mobility matrix would also arise in confined geometries. For example, dense colloids would sediment close to a bottom wall and then diffusive primarily in the plane of the wall. Similarly, colloids may be confined to diffuse in a plane by two walls in a slit channel. The anomalous short-time collective diffusion we studied here will be important in these types of wall-bounded systems as well. In fact, a reanalysis of earlier experiments [3] has shown anomalous behavior of the collective diffusion coefficient with wavenumber in several experimental realizations of quasi one- and twodimensional systems. The confinement by the walls screens the hydrodynamic interactions and will therefore reduce the enhancement of the collective diffusion. For particles confined to diffuse above a single no-slip wall, the hydrodynamic interactions decay like the cubed of the distance, but, counter-intuitively, the interactions decay like the square of the distance for particles in a slit channel [59]. In both cases the compressibility of the hydrodynamic kernel in the plane of diffusion is nonzero. Future work should explore the consequences of this for collective diffusion both with simulations and experiments. The hydrodynamic kernel can easily be changed in our two-dimensional BD-HI algorithm to take into account the Green's function for Stokes flow in confined geometries; however, even for particles diffusing strictly in a plane the method requires an uncontrolled approximation. Specifically, when confinement breaks the symmetry between the two half spaces bounded by the plane of diffusion, forces parallel to the plane can induce motion perpendicular to the plane; a similar effect appears for diffusion on curved interfaces as well. This effect can be taken into account but at a significant increase in complexity. Instead, it may be more appropriate (and physically realistic) to use three-dimensional Brownian dynamics resolving the confining potential, at least for the case of particles sedimented above a bottom wall [60].

\section{Acknowledgments}

We thank Eric Vanden-Eijnden and Johannes Bleibel for informative discussions. This work was supported in part by the National Science Foundation under collaborative award DMS-1418706 and by DMS-1418672, and by the U.S. Department of Energy Office of Science, Office of Advanced Scientific Computing Research, Applied Mathematics program under award DE-SC0008271. We thank the NVIDIA Academic Partnership program for providing GPU hardware for performing some of the simulations reported here. R.D-B and 
SP acknowledge the support of the Spanish Ministry of Science and Innovation MINECO (Spain) under grant FIS2013-47350-C5-1-R and the "María de Maeztu" Programme for Units of Excellence in R\&D (MDM-2014-0377). Part of the simulations were done in Marenostrum under grant FI-2017-2-0023. R.D-B and RP acknowledges support the donors of The American Chemical Society Petroleum Research Fund for partial support of this research via PRF-ACS ND9 grant.

[1] Gerald G Fuller and Jan Vermant. Complex fluid-fluid interfaces: rheology and structure. Annual review of chemical and biomolecular engineering, 3:519-543, 2012.

[2] Frank L. H. Brown. Continuum simulations of biomembrane dynamics and the importance of hydrodynamic effects. Quarterly Reviews of Biophysics, 44(04):391-432, 2011.

[3] Binhua Lin, Bianxiao Cui, Xinliang Xu, Ronen Zangi, Haim Diamant, and Stuart A Rice. Divergence of the long-wavelength collective diffusion coefficient in quasi-one-and quasi-twodimensional colloidal suspensions. Physical Review E, 89(2):022303, 2014.

[4] Colm P Kelleher, Anna Wang, Guillermo Iván Guerrero-García, Andrew D Hollingsworth, Rodrigo E Guerra, Bhaskar Jyoti Krishnatreya, David G Grier, Vinothan N Manoharan, and Paul M Chaikin. Charged hydrophobic colloids at an oil-aqueous phase interface. Physical Review E, 92(6):062306, 2015.

[5] Joseph R Samaniuk and Jan Vermant. Micro and macrorheology at fluid-fluid interfaces. Soft matter, 10(36):7023-7033, 2014.

[6] RB Jones, BU Felderhof, and JM Deutch. Diffusion of polymers along a fluid-fluid interface. Macromolecules, 8(5):680-684, 1975.

[7] Alvaro Domínguez, P Malgaretti, MN Popescu, and S Dietrich. Collective dynamics of chemically active particles trapped at a fluid interface. Soft matter, 12(40):8398-8406, 2016.

[8] Johannes Bleibel, A Domínguez, F Günther, J Harting, and M Oettel. Hydrodynamic interactions induce anomalous diffusion under partial confinement. Soft matter, 10(17):2945-2948, 2014 .

[9] Johannes Bleibel, Alvaro Domínguez, and Martin Oettel. A dynamic dft approach to generalized diffusion equations in a system with long-ranged and hydrodynamic interactions. Journal of Physics: Condensed Matter, 28(24):244021, 2016. 
[10] Johannes Bleibel, Alvaro Domínguez, and Martin Oettel. 3d hydrodynamic interactions lead to divergences in 2d diffusion. Journal of Physics: Condensed Matter, 27(19):194113, 2015.

[11] Binhua Lin, Stuart A Rice, and DA Weitz. Experimental evidence for the divergence of a transport coefficient in a quasi-two-dimensional fluid. Physical Review E, 51(1):423, 1995.

[12] Raphaël Pesché and Gerhard Nägele. Stokesian dynamics study of quasi-two-dimensional suspensions confined between two parallel walls. Physical Review E, 62(4):5432, 2000.

[13] S. Panzuela, Raúl P. Peláez, and R. Delgado-Buscalioni. Collective colloid diffusion under soft two-dimensional confinement. Phys. Rev. E, 95:012602, 2017.

[14] J. Bleibel, Alvaro Domínguez, and M. Oettel. Onset of anomalous diffusion in colloids confined to quasimonolayers. Phys. Rev. E, 95:032604, 2017.

[15] A. Donev and E. Vanden-Eijnden. Dynamic Density Functional Theory with hydrodynamic interactions and fluctuations. J. Chem. Phys., 140(23):234115, 2014.

[16] A. Donev, T. G. Fai, and E. Vanden-Eijnden. A reversible mesoscopic model of diffusion in liquids: from giant fluctuations to Fick's law. Journal of Statistical Mechanics: Theory and Experiment, 2014(4):P04004, 2014.

[17] Andrew J Archer and Markus Rauscher. Dynamical density functional theory for interacting brownian particles: stochastic or deterministic? Journal of Physics A: Mathematical and General, 37(40):9325, 2004.

[18] J. M. O. De Zarate and J. V. Sengers. Hydrodynamic fluctuations in fluids and fluid mixtures. Elsevier Science Ltd, 2006.

[19] A. Vailati, R. Cerbino, S. Mazzoni, C. J. Takacs, D. S. Cannell, and M. Giglio. Fractal fronts of diffusion in microgravity. Nature Communications, 2:290, 2011.

[20] A. Donev R. Cerbino, Y. Sun and A. Vailati. Dynamic scaling for the growth of non-equilibrium fluctuations during thermophoretic diffusion in microgravity. Scientific Reports, 5, 2015. Software available at https://github.com/stochasticHydroTools/MixingIBAMR.

[21] Zhiyuan Qi, Cheol Soo Park, Matthew A. Glaser, Joseph E. Maclennan, and Noel A. Clark. Experimental realization of an incompressible newtonian fluid in two dimensions. Phys. Rev. E, 93:012706, 2016.

[22] Doriano Brogioli and Alberto Vailati. Nonequilibrium fluctuations during diffusion in liquid layers. Phys. Rev. E, 96:012136, 2017.

[23] Tatiana Kuriabova, Thomas R Powers, Zhiyuan Qi, Aaron Goldfain, Cheol Soo Park, 
Matthew A Glaser, Joseph E Maclennan, and Noel A Clark. Hydrodynamic interactions in freely suspended liquid crystal films. Physical Review E, 94(5):052701, 2016.

[24] Vincent Demery, Olivier Benichou, and Hugo Jacquin. Generalized Langevin equations for a driven tracer in dense soft colloids: Construction and applications. New Journal of Physics, $16,2014$.

[25] David C Morse. Theory of constrained brownian motion. Advances in Chemical Physics, 128(65-189):110, 2004.

[26] G. Ciccotti, T. Lelièvre, and E. Vanden-Eijnden. Projection of diffusions on submanifolds: Application to mean force computation. Communications on Pure and Applied Mathematics, 61(3):371-408, 2008.

[27] Ibrahim Fatkullin, Gregor Kovacic, Eric Vanden-Eijnden, et al. Reduced dynamics of stochastically perturbed gradient flows. Communications in Mathematical Sciences, 8(2):439-461, 2010 .

[28] H. C.Öttinger. Preservation of thermodynamic structure in model reduction. Phys. Rev. E, 91:032147, 2015.

[29] Jens Rotne and Stephen Prager. Variational treatment of hydrodynamic interaction in polymers. The Journal of Chemical Physics, 50:4831, 1969.

[30] F. Balboa Usabiaga, X. Xie, R. Delgado-Buscalioni, and A. Donev. The Stokes-Einstein Relation at Moderate Schmidt Number. J. Chem. Phys., 139(21):214113, 2013.

[31] M Rex and H Löwen. Dynamical density functional theory for colloidal dispersions including hydrodynamic interactions. The European Physical Journal E, 28(2):139-146, 2009.

[32] A. Donev, E. Vanden-Eijnden, A. L. Garcia, and J. B. Bell. On the Accuracy of FiniteVolume Schemes for Fluctuating Hydrodynamics. Communications in Applied Mathematics and Computational Science, 5(2):149-197, 2010.

[33] F. Balboa Usabiaga, R. Delgado-Buscalioni, B. E. Griffith, and A. Donev. Inertial Coupling Method for particles in an incompressible fluctuating fluid. Comput. Methods Appl. Mech. Engrg., 269:139-172, 2014. Code available at https://github.com/fbusabiaga/fluam.

[34] S. Delong, F. Balboa Usabiaga, R. Delgado-Buscalioni, B. E. Griffith, and A. Donev. Brownian Dynamics without Green's Functions. J. Chem. Phys., 140(13):134110, 2014. Software available at https://github.com/stochasticHydroTools/FIB.

[35] Eric E. Keaveny. Fluctuating force-coupling method for simulations of colloidal suspensions. 
J. Comp. Phys., 269(0):61 - 79, 2014.

[36] Blaise Delmotte and Eric E Keaveny. Simulating brownian suspensions with fluctuating hydrodynamics. The Journal of chemical physics, 143(24):244109, 2015.

[37] A. M. Fiore, F. Balboa Usabiaga, A. Donev, and J. W. Swan. Rapid sampling of stochastic displacements in brownian dynamics simulations. J. Chem. Phys., 146(12):124116, 2017. Software available at https://github.com/stochasticHydroTools/PSE.

[38] M. R. Maxey and B. K. Patel. Localized force representations for particles sedimenting in Stokes flow. International journal of multiphase flow, 27(9):1603-1626, 2001.

[39] P. J. Atzberger. Stochastic Eulerian-Lagrangian Methods for Fluid-Structure Interactions with Thermal Fluctuations. J. Comp. Phys., 230:2821-2837, 2011.

[40] P. Español and A. Donev. Coupling a nano-particle with isothermal fluctuating hydrodynamics: Coarse-graining from microscopic to mesoscopic dynamics. J. Chem. Phys., 143(23), 2015 .

[41] H Hasimoto. On the periodic fundamental solutions of the stokes equations and their application to viscous flow past a cubic array of spheres. J. Fluid Mech, 5(02):317-328, 1959.

[42] Y. Bao, M. Rachh, E. Keaveny, L. Greengard, and A. Donev. A fluctuating boundary integral method for Brownian suspensions. Submitted to J. Comp. Phys., preprint ArXiv:1709.01480, 2017.

[43] L. Greengard and J. Lee. Accelerating the nonuniform fast fourier transform. SIAM Review, 46(3):443-454, 2004.

[44] D. Frenkel and B. Smit. Understanding Molecular Simulation. Academic Press, 2002.

[45] M Rex and H Löwen. Dynamical density functional theory with hydrodynamic interactions and colloids in unstable traps. Phys. Rev. Lett., 101(14):148302, 2008.

[46] RJA Tough, PN Pusey, HNW Lekkerkerker, and C Van Den Broeck. Stochastic descriptions of the dynamics of interacting brownian particles. Molecular Physics, 59(3):595-619, 1986.

[47] David S Dean. Langevin equation for the density of a system of interacting langevin processes. Journal of Physics A: Mathematical and General, 29(24):L613, 1996.

[48] S. Delong, Y. Sun, B. E. Griffith, E. Vanden-Eijnden, and A. Donev. Multiscale temporal integrators for fluctuating hydrodynamics. Phys. Rev. E, 90:063312, 2014. Software available at https://github.com/stochasticHydroTools/MixingIBAMR.

[49] F. Croccolo, D. Brogioli, A. Vailati, M. Giglio, and D. S. Cannell. Nondiffusive decay of 
gradient-driven fluctuations in a free-diffusion process. Phys. Rev. E, 76(4):041112, 2007.

[50] J.A. de la Torre, P. Español, and A. Donev. Finite element discretization of non-linear diffusion equations with thermal fluctuations. J. Chem. Phys., 142(9):094115, 2015.

[51] C. Kim, A. J. Nonaka, A. L. Garcia, J. B. Bell, and A. Donev. Stochastic simulation of reaction-diffusion systems: A fluctuating-hydrodynamics approach. J. Chem. Phys., 146(12), 2017. Software available at https://github.com/BoxLib-Codes/FHD_ReactDiff.

[52] David S Dean and Vincent Démery. Diffusion of active tracers in fluctuating fields. Journal of Physics: Condensed Matter, 23(23):234114, 2011.

[53] Vincent Démery and David S Dean. Perturbative path-integral study of active-and passivetracer diffusion in fluctuating fields. Physical Review E, 84(1):011148, 2011.

[54] Umberto Marini Bettolo Marconi and Pedro Tarazona. Dynamic density functional theory of fluids. The Journal of Chemical Physics, 110(16):8032-8044, 1999.

[55] Pep Español and Hartmut Löwen. Derivation of dynamical density functional theory using the projection operator technique. J. Chem. Phys., 131:244101, 2009.

[56] PG Saffman. Brownian motion in thin sheets of viscous fluid. Journal of Fluid Mechanics, 73(04):593-602, 1976.

[57] Naomi Oppenheimer and Haim Diamant. Correlated diffusion of membrane proteins and their effect on membrane viscosity. Biophysical Journal, 96(8):3041-3049, 2009.

[58] Brian A. Camley, Michael G. Lerner, Richard W. Pastor, and Frank L. H. Brown. Strong influence of periodic boundary conditions on lateral diffusion in lipid bilayer membranes. The Journal of Chemical Physics, 143(24):243113, 2015.

[59] Haim Diamant. Hydrodynamic interaction in confined geometries. Journal of the Physical Society of Japan, 78(4):041002, 2009.

[60] Florencio Balboa Usabiaga, Blaise Delmotte, and Aleksandar Donev. Brownian dynamics of confined suspensions of active microrollers. J. Chem. Phys., 146(13):134104, 2017. Software available at https://github.com/stochasticHydroTools/RigidMultiblobsWall. 